\title{
Exchange of medicinal plant information in California missions
}

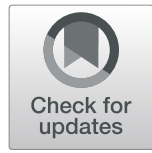

Joe Rayl McBride ${ }^{1 *} \mathbb{D}$, Rita Yolanda Cavero ${ }^{2}$, Anna Liisa Cheshire $^{3}$, María Isabel Calvo ${ }^{4}$ and Deborah Lea McBride ${ }^{5}$

\begin{abstract}
Background: Missions were established in California in the eighteenth and nineteenth centuries to convert Native Americans to Christianity and enculturate them into a class of laborers for Californios (Spanish/Mexican settler). The concentration of large numbers of Native Americans at the Missions, along with the introduction of European diseases, led to serious disease problems. Medicinal supplies brought to California by the missionaries were limited in quantity. This situation resulted in an opportunity for the sharing of knowledge of medicinal plants between the Native Americans and the Mission priests. The purpose of this study is to examine the degree to which such sharing of knowledge took place and to understand factors that may have influenced the sharing of medicinal knowledge. The study also examines the sharing of medicinal knowledge between the Native Americans and the Californios following the demise of the California Missions.

Methods: Two methods were employed in the study: (1) a comparison of lists of medicinal plants used by various groups (e.g., Native American, Mission priests, Californios) prior to, during, and after the Mission period and (2) a close reading of diaries, reports, and books written by first-hand observers and modern authorities to find accounts of and identify factors influencing the exchange of medicinal information.

Results: A comparison of the lists of medicinal plants use by various groups indicated that only a small percentage of medicinal plants were shared by two or more groups. For example, none of the 265 taxa of species used by the Native Americans in pre-Mission times were imported into Spain for medicinal use and only 16 taxa were reported to have been used at the Missions. A larger sharing of information of medicinal plants took place in the post-Mission period when Native Americans were dispersed from the Missions and worked as laborers on the ranches of the Californios.

*Correspondence: jrm2@berkeley.edu

'Department of Environmental Science, Policy and Management, University of California, Berkeley, CA, USA

Full list of author information is available at the end of the article

(c) The Author(s). 2020 Open Access This article is licensed under a Creative Commons Attribution 4.0 International License, which permits use, sharing, adaptation, distribution and reproduction in any medium or format, as long as you give appropriate credit to the original author(s) and the source, provide a link to the Creative Commons licence, and indicate if changes were made. The images or other third party material in this article are included in the article's Creative Commons licence, unless indicated otherwise in a credit line to the material. If material is not included in the article's Creative Commons licence and your intended use is not permitted by statutory regulation or exceeds the permitted use, you will need to obtain permission directly from the copyright holder. To view a copy of this licence, visit http://creativecommons.org/licenses/by/4.0/. The Creative Commons Public Domain Dedication waiver (http://creativecommons.org/publicdomain/zero/1.0/) applies to the data made available in this article, unless otherwise stated in a credit line to the data. 


\begin{abstract}
(Continued from previous page)
Conclusions: Sharing of information concerning medicinal plants did occur during the Mission period, but the number of documented species was limited. A number of possible factors discouraged this exchange. These include (1) imbalance of power between the priests and the Native Americans, (2) suppression of indigenous knowledge and medical practices by the Mission priests, (3) language barriers, (4) reduction of availability of medicinal herbs around the Mission due to introduced agricultural practices, (5) desire to protect knowledge of medicinal herbs by Native American shaman, (6) administrative structure at the Missions which left little time for direct interaction between the priests and individual Native Americans, (7) loss of knowledge of herbal medicine by the Native Americans over time at the Missions, and (8) limited transportation opportunities for reciprocal the shipment of medicinal plants between California and Spain. Three possible factors were identified that contributed to a greater sharing of information between the Native Americans and the Californios in the post-Mission period. These were (1) more one-to-one interactions between the Californios and the Native Americans, (2) many of the Californios were mestizos whose mothers or grandmothers were Native Americans, and (3) lack of pressure on the part of the Californios to suppress Native American beliefs and medicinal practices.
\end{abstract}

Keywords: Medicinal plants, Native Americans, California Missions, Spanish priests, Information transfer, Californios

\section{Background}

The migration of people to North America began about 21,000-40,000 years BP over a great land bridge between Siberia and Alaska [1]. Evidence of human settlement dates from about 13,000 years BP on the Channel Islands off the coast of California and from about 10,330 years BP on the mainland near San Luis Obispo ([2]). These early immigrants moved along a coastal route from Alaska either on foot or by boat. Later, Native Americans immigrated to coastal California from inland California and from more eastern areas of North America. They brought with them about 100 languages belonging to seven major language groups [3]. The immigrants also brought with them knowledge of plants used for medicinal purposes gained from the territories they had previously occupied. For example, roots of the species in genus Rubus (blackberries) were used to control diarrhea by people in Asia as well as by Native Americans living in different parts of North America [4]. When people immigrated to California, they adopted local species of Rubus to combat diarrhea [5]. Once in California, the immigrants adapted new species for medicinal use. The Pomo, for example, used the bark of the California buckeye (Aesculus californica), a California endemic, to treat snakebites [6]. Various researchers have examined medicinal use of plants by Native Americans in California since the nineteenth century [719]. These studies served as important references in the study reported here.

The culture and economy of Native Americans was changed significantly beginning in 1769 with the European colonization of California. An integral part of the Spanish colonization process was the establishment of a system of Missions (Fig. 1). The first Mission was located in what was to become the city of San Diego. Subsequently, Franciscan priests supported by the military moved northward along the California coast to establish a total of 21 Missions [20]. These Missions were established to christianize the Native Americans and to prepare them to serve as a peasant class in the new Spanish territory [21].

In the early Mission period, the priests staffing the Missions were mostly from Spain. The Franciscan priests who established and staffed Missions came primarily from Spain [22, 23]. Thirty-six (72\%) of the priests came from northern Spain (Basque territory and the adjacent provinces, Navarra mainly), one from central Spain (2\%), and none from the south of Spain. The remaining priests were from Mallorca (8 individuals, 16\%) and Mexico (5 individuals, 10\%). The Basque territory, Navarra, and Mallorca were the homelands of one-half of the priest at the early California Missions. These priests brought with them knowledge of medicinal herbs used in their homelands. They also brought seeds and cuttings of plants [21] used for medicinal purposes in Mexico and Spain [24].

The California Missions were under the control of Spain from 1769 to 1821 . During this time the Native Americans who were converted to christianity at the Missions were known to as neophytes. The medical care of the neophytes was one of the responsibilities of the priests. The neophytes, not being immune to European diseases, succumbed in large numbers to epidemics of measles and smallpox [25]. Contagious native ailments (e.g., colds, dysentery) also spread among the neophytes due to their congregation in large numbers at the Missions. The priests responded to the increasing numbers of sick neophytes by establishing hospitals at many of the Missions. Although there was a significant power imbalance between the priests and the neophytes, the situation called for a sharing of information about medicinal herbs and the employment of neophytes in the treatment of the sick. Enfermeros (neophytes selected by the priests to serve as nurses) were assigned to care for the sick in these hospitals. The enfermeros used medicinal herbs and Spanish medicine to treat the neophytes. Medicinal 


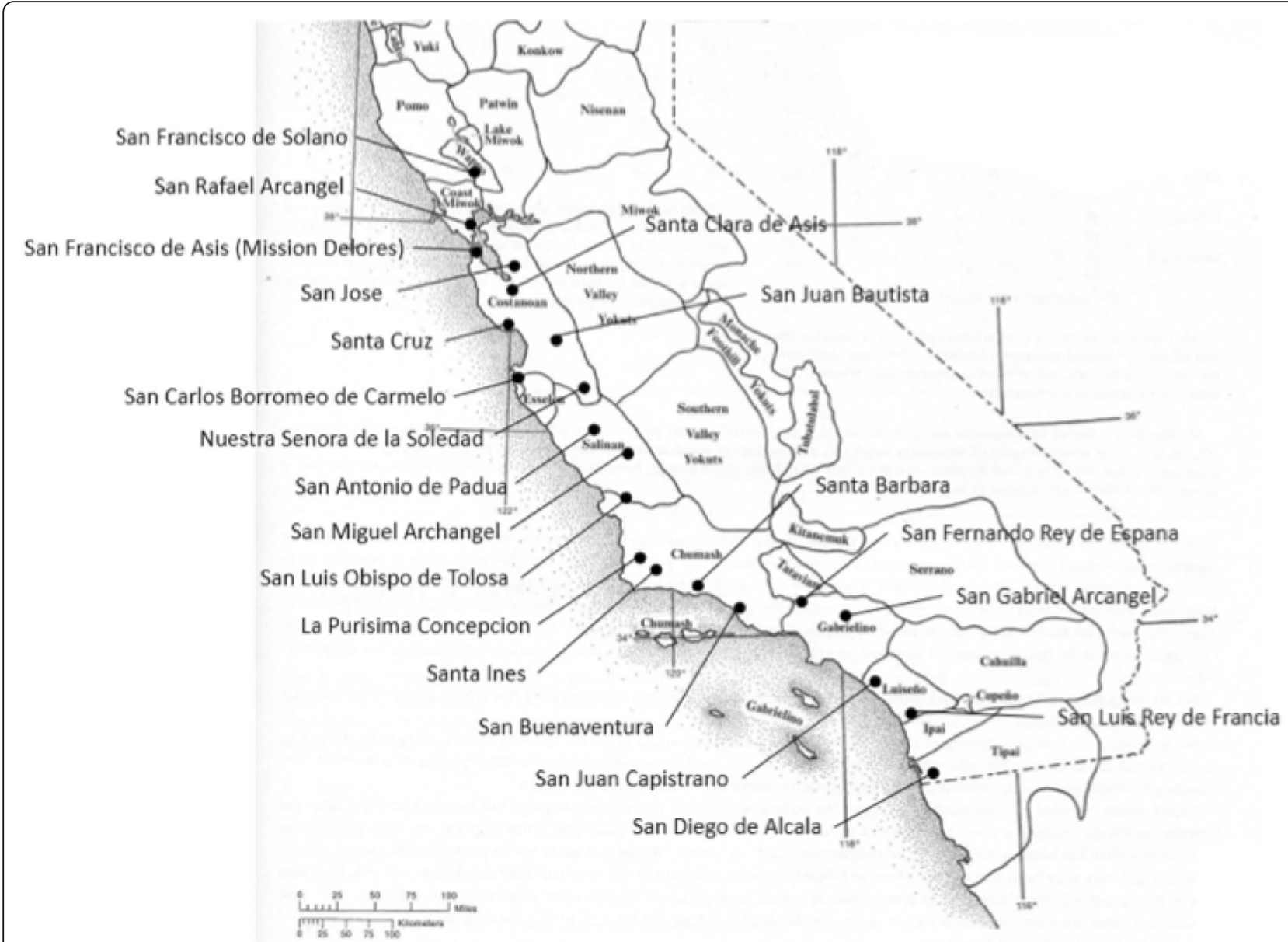

Fig. 1 Locations of California Missions and Native American tribal territories

herbs used by the Native Americans were collected from around the Missions [21], while Spanish medicinal supplies were shipped periodically to California from Mexico [7]. The quantity of medicinal supplies imported from Mexico often became inadequate to treat the increasing number of neophytes succumbing to both native and exotic diseases. At times of shortages of medical supplies, the priests and enfermeros exchanged knowledge of medicinal plants to broaden the supply of medicines to treat the sick [26]. Neophytes were sometimes dispatched by the priests to collect medicinal plants from the wild (Engelhardt 1922).

During the Mission period, seeds of plants for the mission gardens periodically arrived via ships from Europe, South America, and Mexico. Walled gardens, known as huertas, were an essential part of the Mission landscapes. They provided growing space for food plants, as well as trees, flowers, and medicinal herbs. Plants grown in the huertas were used by both the priests and the Native Americans. The importation of seeds and other goods was curtailed after 1810 when shipping from Spain and the Spanish colonies in the New World was interrupted by the rebellion in Mexico [21]. Mexico gained its independence from Spain in 1821. Following the Mexican rebellion, the independent Mexican government exerted its authority over the Missions. The Mexican authorities attempted to expel the Franciscan priests from the Missions, sell or transfer Mission lands to Mexican citizens, and convert the Mission churches to local parish churches. This process was known as "secularization." Some missions were abandoned while others assumed the role of parish churches. Mission in more remote locations in California still housed limited number of Native American neophytes, but most neophytes were transferred to nearby ranches during the Mexican period (1821-1848) were they worked as laborers. Some Native Americans were paid modest salaries for their labor, while most worked for food and a place to live. Individual Native American families and extended families lived on the ranches. A striking contrast to the hundreds who had resided at the missions. The relocation of Native Americans to local ranches provided an opportunity for the sharing 
of information concerning medicinal plants between the Native Americans and the Californios.

The secularization period ended in 1848 with the annexation of California by the USA following the war with Mexico. Following the annexation, most of the Missions were abandoned and began to fall into disrepair. Without active parishes to maintain the Missions, the old buildings fell prey to the weather. Their roofs gave way first, exposing the soluble adobe walls to the rain. Many of the old buildings were abandoned as unsafe or unsalvageable, many were torn down. For many decades the decay of buildings at the Missions, the missions continued until citizens began to take an interest in them and to propose their restoration. Old records, drawing, and photographs were studied to perform reconstruction of historic buildings, patios, and gardens. At several Missions, medicinal plants were incorporated into the restored gardens.

The purpose of this study is to examine the exchange of medicinal plant information at the California Missions during the Mission and post-Mission periods. Specifically, the exchange between the Native Americans and the priests during the Mission period and the exchange between the Native Americans and the Californios during and following the secularization of the Missions. We hypothesize that an exchange of information on medicinal plants can be identified by comparing the numbers of taxa from Spain that were introduced into California and adopted for use by the Native Americans and the number of taxa from California that were introduced into Spain and adopted by Spanish citizens for medicinal purposes. Furthermore, the exchange of information concerning medicinal plants between the Native Americans and the Californios can be identified by the number of medicinal taxa from Spain and Mexico that were introduced into California and used by the Native Americans and the number of California taxa adopted for medicinal use by the Californios.

\section{Methods}

Two methods were employed in this study: (1) comparison of lists of medicinal plants used by Native American in California before the Mission period, medicinal plants used in Spain, medicinal plants used in Mexico before it gained its independence from Spain, and medicinal plants used by Californios and Native Americans in the post-Mission period and (2) a close reading of diaries, journals, reports, and books written by (i) first-hand observers during the Mission and post-Mission periods and, (ii) modern anthropologists, ethnobotanists, and historians to find accounts of the sharing of information about medicinal plants and to identify reasons why an exchange of information may or may not have taken place.
The lists of medicinal plants and their uses were assembled from a number of sources (Table 1) for the pre- and Mission period (before and during colonization) and the post Mission Period (during and after secularization).

The data provided were grouped into 14 categories depending on the pathology they treated [37, 38, 44]: (1) cardiovascular diseases; (2) depurative; (3) dermatology; (4) digestive or gastrointestinal problems; (5) metabolic syndromes; (6) infections; (7) skeleto-muscular system; (8) nervous system; (9) sens (eye and ear problems); (10) gynecology; (11) respiratory complaints; (12) urology; (13) ritual procedures; (14) various other ailments (Table 2). Botanical family classification and nomenclature for species names were authenticated according to Hickman [45], Stevens [46] and [47] (www.ipni.org).

To determine if any California species were introduced in Spanish and/or European botanical gardens a literature review was carried conducted [48-54]. Several databases were also consulted: www.floraiberica.es; www. fitoterapia.net [55-57];

A comparison of the assembled lists identified medicinal plant taxa that were used in two different areas (e.g., California and Spain). If taxa native to California were reported to be used in present-day Spanish medicinal gardens, then we assumed information of the medicinal use of these plants had been shared between the Native Americans and the Spanish priest. Likewise, if taxa native to Spain were present in herb gardens at the Missions or reported to have been used by Native

Table 1 Bibliographic sources used to assemble the lists of medicinal plants used in different areas

\begin{tabular}{ll}
\hline Area & Source \\
\hline California (Native Americans) & Barrows [27] \\
& Bean and Saubel [8] \\
& Faber and Lasagna [28] \\
& Heinsen [29] \\
& Lightfoot and Parrish [30] \\
& Mead [31] \\
& Timbrook [18] \\
& Wilken-Robertson [32] \\
& Akerreta et al. [33, 34] \\
& Alarcón et al. [35] \\
& Carrió and Vallès [36] \\
Spain & Cavero et al. [37, 38] \\
& Menendez-Baceta et al. [39] \\
& Argueta and Gallardo [40] \\
& Heinrich et al. [41] \\
Simpson [42] & Beebe and Senkewicz [43] \\
Mexico (Viceroyalty of New Spain) & Weber [19] \\
\hline
\end{tabular}


Table 2 Classifying diseases

\begin{tabular}{|c|c|c|}
\hline Number & Categories & Affection \\
\hline 1 & CAR: Cardiovascular diseases & $\begin{array}{l}\text { Antivaricose, blood disorders, blood pressure regulator (thick blood, antihypertensive), cardiotonic } \\
\text { (heart problems), clean the blood, external hemostatic, hemorrhoids (piles), high cholesterol, } \\
\text { phlebitis, uric acid, vasotonic (circulatory problems, enhance circulation) }\end{array}$ \\
\hline 2 & DEP: Diuretic, laxative, diaphoretic & Clean the body, depurative, fluid retention \\
\hline 3 & DER: Dermatology & $\begin{array}{l}\text { Acne, anti-ecchymotic, baldness (hair loss), bites (dog, snake, insect, nettle stings), blisters and } \\
\text { grazes, boils; bruises, burns, calcanean spurs, calluses or corns, cellulitis, chilblains, clean the skin, } \\
\text { eczema, embedded thorns, gangrene, hard skin, mouth infections and ulcers, pruritus, psoriasis, } \\
\text { skin disorders (infection, inflammation, rash), ulcers; vulnerary, warts, whitlows, wounds and cuts } \\
\text { (infection) }\end{array}$ \\
\hline 4 & $\begin{array}{l}\text { GAS: Digestive or gastrointestinal } \\
\text { problems }\end{array}$ & $\begin{array}{l}\text { Antiemetic, antihelminthic, appetizer (tonic), carminative (gases), clean the stomach, constipation } \\
\text { (laxative), diarrhea, digestive disorders, emetic, gall stones, gastritis (gastric anti-inflammatory), } \\
\text { heartburn, internal ulcers, intestinal worms, liver disorders (clean, inflammation, jaundice, } \\
\text { protection, pain), purgative, stomach pain and disorders, teeth (disorders, strengthening, pain) }\end{array}$ \\
\hline 5 & MET: Metabolic syndromes & $\begin{array}{l}\text { Allergic reactions, anti-inflammatory, diabetes, hypoglycemic, metabolic disorders, salutiferous, } \\
\text { stimulate immune system }\end{array}$ \\
\hline 6 & INF: Infections & Antiherpes, fever (antipyretic), internal antiseptic (infections) \\
\hline 7 & SKE: Skeleto-muscular system & $\begin{array}{l}\text { Antialgic muscular, antispasmodic, arthrosis, body pains, broken bones, decalcifications, lumbago, } \\
\text { muscle anti-inflammatory, muscular and joint pains, musculoskeletal disorders, osteoarthritis } \\
\text { (arthritis), rheumatism (antirheumatic), sciatica, sprains. }\end{array}$ \\
\hline 8 & NER: Nervous system & $\begin{array}{l}\text { Analgesic, antiparkinsonian, depression, headache, insomnia, nervousness, relaxant, sadness, } \\
\text { sedative (tranquilizer), sickness, stimulant }\end{array}$ \\
\hline 9 & SEN: Sens & $\begin{array}{l}\text { Eyes (clean, conjunctivitis, antiseptic, inflammation, irritation, pain, rheum, sties, visual protector), } \\
\text { ear (disorders and pain) }\end{array}$ \\
\hline 10 & GYN: Gynecology & $\begin{array}{l}\text { Abortive, dysmenorrhea, anti-metrorrhagic, emmenagogue, galactofugue, galactogenous, } \\
\text { menstruation, premenstrual pain, puerperium antiseptic, tonic after give birth, vaginal infections. }\end{array}$ \\
\hline 11 & RES: Respiratory complaints & $\begin{array}{l}\text { Anticatarrhal, antitussive, asthma, bronchitis, chest infections, cold, cough, expectorant } \\
\text { (mucolytic) flu, hoarseness, inflammation, influenza, pharyngeal problems, phlegm; pneumonia, } \\
\text { sinusitis, sore throat, tuberculosis, whooping cough }\end{array}$ \\
\hline 12 & URO: Urology & $\begin{array}{l}\text { Cystitis, kidney disorders (stones and clean), masculine impotence, prostate inflammations and } \\
\text { disorders, renal anti-inflammatory, litothriptic and protector, urinary antiseptic and retention }\end{array}$ \\
\hline 13 & RIT: Ritual procedures & To protect from illness and bad spirits \\
\hline 14 & VAR: Various & $\begin{array}{l}\text { Undefined pain and illnesses (anemia, antiscorbutic, diaphoretic, general malaise and pains, } \\
\text { healthy, iron- deficiency, panacea, to give up alcohol, and vitamin) }\end{array}$ \\
\hline
\end{tabular}

Americans during the post-Mission period, we assumed that sharing of knowledge had taken place.

\section{Results}

A total of 822 taxa belonging to 136 botanical families were identified (Table 3). Seven hundred twelve of them had been used during pre- and Mission Period; 265 of them were plants used by Native Americans in California before colonization, 448 taxa were used for medicinal purposes in Spain or in Mexico (Table 3). The most commonly used plants were employed to treat sores, wounds, and skin problems, for respiratory diseases, gastrointestinal tract problems, reproductive affections, and cardiovascular diseases (Fig. 2). The preparation and application of plant materials for medicinal purposes by the Native Americans in California included the direct application of leaves to the affected area (e.g., Rhamnus californica Eschsch.-treat rheumatism); drinking water in which the plant material had been boiled (e.g., Rubus ursinus Cham. \& Schldl-treat diarrhea); application of a poultice prepared from the plant material (e.g., Malva parviflora L.- treat wounds), eating the plant or plant part (e.g., Rorippa nasturtium (L.) Hayek-treat liver ailments), bathing the skin with water in which to plant had been boiled (e.g., Wyethia helenioides (DC.) Nutt.treat sores); rubbing dry ashes of a plant on the skin (e.g., Scripus californicus (C. Mewyer) Steudel-treat poison oak); chewing plant parts (e.g., Lomatium californicum (Torrey and Gray) J. Coulter \& Rose-treat pain).

We assumed if information concerning California medicinal plants was shared by the Native Americans with the Spanish priests some of these species would have been subsequently introduced to Spain as had medicinal plants from Mexico and South America.

Twelve of 265 taxa used by Native Americans were also used in Mexico: Adiantum aleuticum (Rupr.) C.A. Paris, Anemopsis californica (Nutt.) Hook. \& Arn, Artemisia ludoviciana Nutt, Baccharis glutinosa Pers., Cucurbita foetidissima Kunth, Equisetum arvense L., Larrea tridentata (DC.) Cov., Opuntia sp., Quercus sp., 
Table 3 Medicinal plants used before, during and after the Mission period, and present time at Mission Gardens. The numbers refer to emic and etic illness groupings (see Table 2)

\begin{tabular}{|c|c|c|c|c|c|}
\hline Botanical family* & Medicinal plants* & Native & $\begin{array}{l}\text { Pre-Mission } \\
\text { period }\end{array}$ & Mission period & $\begin{array}{l}\text { Post- } \\
\text { Mission } \\
\text { period }\end{array}$ \\
\hline \multirow[t]{2}{*}{ Acanthaceae } & Acanthus mollis L. & Europe & & 3 & \\
\hline & Justicia spicigera Schltdl. & Mexico & & 3,4 & \\
\hline \multirow[t]{4}{*}{ Adoxaceae } & Sambucus ebulus L. & Eurasia & & 3 & \\
\hline & Sambucus sp. & California & 3,11 & & $3,6,10$ \\
\hline & $\begin{array}{l}\text { Sambucus mexicana C. Presl [S. nigra } \\
\text { L. ssp. caerulea (Raf.) R. Bolli] }\end{array}$ & California, Mexico & $\begin{array}{l}1,3,4,6,7,8 \\
10,11\end{array}$ & 7,10 & 8,11 \\
\hline & Sambucus nigra L. ssp. nigra & Europe, Africa & & $\begin{array}{l}1,2,3,4,6,7,8 \\
9,10,11,12\end{array}$ & \\
\hline \multirow[t]{2}{*}{ Agavoideae } & Agave sp. & California and Mexico & & 4 & \\
\hline & Agave americana L. & $\begin{array}{l}\text { Mexico, USA introduced } \\
\text { from Europe }\end{array}$ & & 11 & \\
\hline \multirow[t]{12}{*}{ Amaranthaceae } & Amaranthus hybridus L. & $\begin{array}{l}\text { Eastern U.S.A. introduced } \\
\text { from Europe }\end{array}$ & & $1,3,4$ & \\
\hline & Atriplex sp. & California & 10 & & \\
\hline & Atriplex lentiformis (Torrey) S. Watson & California & $3,9,10$ & & \\
\hline & Beta vulgaris L. var. conditiva Alef. & Eurasia, Africa & & 1 & \\
\hline & Beta vulgaris L. var. maritima (L.) Moq. & Eurasia, Africa & & 5 & \\
\hline & $\begin{array}{l}\text { Chenopodium ambrosioides L. [Dysohania } \\
\text { ambrosioides (L.) Mosyakin \& Clemants] }\end{array}$ & Mexico & & 4 & 3 \\
\hline & $\begin{array}{l}\text { Chenopodium californicum (S. Watson) } \\
\text { S. Watson }\end{array}$ & California & $4,8,10$ & & \\
\hline & Chenopodium graveolens Willd. & Mexico & & 4,10 & \\
\hline & $\begin{array}{l}\text { Chenopodium rubrum L. [Oxybasis rubra } \\
\text { (L.) S. Fuentes, Uotila \& Borsch] }\end{array}$ & California & & & \\
\hline & $\begin{array}{l}\text { Dysphania ambrosioides (L.) Mosyakin } \\
\text { \& Clemants }\end{array}$ & Mexico & & & 3 \\
\hline & $\begin{array}{l}\text { Dysphania botrys (L.) Mosyakin } \\
\text { \& Clemants }\end{array}$ & Europe & & & 3 \\
\hline & Iresine celosia L. & Mexico & & $3,6,12$ & \\
\hline \multirow[t]{4}{*}{ Amaryllidaceae } & Allium sp. & California & $3,4,11$ & & 10,11 \\
\hline & Allium cepa L. & Asia introduced from Europe & & $1,3,4,11,12$ & \\
\hline & Allium porrum L. & Europe & & $1,4,11$ & \\
\hline & Allium sativum $\mathrm{L}$. & Asia introduced from Europe & & $\begin{array}{l}1,3,4,5,6,7,8 \\
11\end{array}$ & $6,11,12$ \\
\hline \multirow[t]{8}{*}{ Anacardiaceae } & Mangifera indica L. & India & & 4,9 & \\
\hline & Pistacia lentiscus L. & Mediterranean region & & 3,4 & \\
\hline & Rhus aromatic L. (R. trilobata Nutt.) & California & 4 & & \\
\hline & Rhus ovate S. Watson & California & 1,10 & & \\
\hline & Schinus molle L. & South America & & $4,6,7,9,10$ & \\
\hline & Spondias purpurea L. & Mexico & & $4,6,9,10$ & \\
\hline & $\begin{array}{l}\text { Toxicodendron diversilobum (Torrey } \\
\& \text { A. Gray) E. Greene }\end{array}$ & California & $1,3,4,6,9$ & & 3 \\
\hline & $\begin{array}{l}\text { Toxicodendron venenosum (S. Watson) } \\
\text { Rydb. var. venenosum (Zigadenus } \\
\text { venenosus S. Watson) }\end{array}$ & California & 3 & & \\
\hline \multirow{3}{*}{$\begin{array}{l}\text { Anacariaceae } \\
\text { Annonaceae }\end{array}$} & Malosma laurina (Nutt.) Abrams & California & 6 & & \\
\hline & Annona cherimola Mill. & South America & & 4,6 & \\
\hline & Annona reticulate Linn. & Mexico & & 3,4 & \\
\hline \multirow[t]{3}{*}{ Apiaceae } & Angelica sp. & California & $3,4,7,8$ & & \\
\hline & Apium graveolens L. & Europe & & $1,2,11,12$ & 4 \\
\hline & Aralia californica S. Watson & California & 3 & & \\
\hline
\end{tabular}


Table 3 Medicinal plants used before, during and after the Mission period, and present time at Mission Gardens. The numbers refer to emic and etic illness groupings (see Table 2) (Continued)

\begin{tabular}{|c|c|c|c|c|c|}
\hline Botanical family* & Medicinal plants* & Native & $\begin{array}{l}\text { Pre-Mission } \\
\text { period }\end{array}$ & Mission period & $\begin{array}{l}\text { Post- } \\
\text { Mission } \\
\text { period }\end{array}$ \\
\hline & Carum carvi L. & $\begin{array}{l}\text { Europe, naturalized in } \\
\text { California }\end{array}$ & & & \\
\hline & Coriandrum sativum L. & $\begin{array}{l}\text { Europe, naturalized in } \\
\text { California }\end{array}$ & & & 8 \\
\hline & Crithmum maritimum L. & Eurasia, Africa & & $2,4,14$ & \\
\hline & Daucus carota L. & Eurasia & & $1,3,9$ & \\
\hline & Daucus pusillus Michaux & California & $1,3,8,10,11$ & & $1,8,10,11$ \\
\hline & Eryngium campestre L. & Eurasia & & 2,10 & \\
\hline & Foeniculum vulgare Mill. & Europe & & $1,2,3,4,10,11$ & 4 \\
\hline & Hedera helix L. & Europe & & $3,4,10$ & 3,10 \\
\hline & $\begin{array}{l}\text { Lomatium californicum (Torrey \& A. } \\
\text { Gray) Mathias \& Constance } \\
\text { (Leptotaenia californicum Nutt.) }\end{array}$ & California & $4,7,8,10,11$ & & \\
\hline & $\begin{array}{l}\text { Lomatium utriculatum (Torrey and } \\
\text { Gray) J. Coulter \& Rose }\end{array}$ & California & & & 11,13 \\
\hline & Petroselinum crispum (Mill.) Fuss & Europe & & $\begin{array}{l}2,3,4,5,8,9 \\
10,14\end{array}$ & 4,13 \\
\hline & Pimpinella anisum $\mathrm{L}$. & $\begin{array}{l}\text { Asia Minor, introduced from } \\
\text { Europe }\end{array}$ & & 4 & \\
\hline & Sanicula arguta J. Coult. \& Rose & California & 5 & & \\
\hline \multirow[t]{6}{*}{ Apocynaceae } & Gonolobus niger (Cav.) R. Br. & Mexico & & 6 & \\
\hline & Nerium oleander $\mathrm{L}$. & Europe & & & \\
\hline & Plumeria rubra L. & Mexico & & $3,4,6$ & \\
\hline & Stemmadenia donnell-smithi Woodson & Europe & & 3 & \\
\hline & Thevetia thevetioides (Kunth) Schum. & Mexico & & 3 & \\
\hline & Vinca difformis Pourr. & Europe & & 10 & \\
\hline Aquifoliaceae & Ilex aquifolium L. & Europe & & 3 & \\
\hline \multirow[t]{2}{*}{ Araceae } & Arisarum vulgare Targ.-Tozz. & Eurasia, Africa & & 3 & \\
\hline & Arum italicum Mill. & Mediterranean region & & 3,7 & \\
\hline \multirow[t]{2}{*}{ Arecaceae } & Chamaerops humilis L. & Europe & & 3,4 & \\
\hline & Cocos nucifera L. & Malaysia & & & 6 \\
\hline \multirow[t]{4}{*}{ Aristolochiaceae } & Aristolochia maurorum L. & Mexico & & 8 & \\
\hline & Aristolochia monticola Brandegee & Mexico & & & 3,4 \\
\hline & Aristolochia pentandra Jacq. & Mexico & & 3 & \\
\hline & Asarum caudatum Lindl. & California & 3,7 & & \\
\hline \multirow[t]{4}{*}{ Asclepiadaceae } & Asclepias sp. & California & 4 & & \\
\hline & Asclepias curassavica $\mathrm{L}$. & Mexico & & 3,9 & 9 \\
\hline & Asclepias eriocarpa Benth. & California & $3,4,10$ & & \\
\hline & Asclepias lemmonii A. Gray & Mexico, South West USA & & & 11 \\
\hline \multirow[t]{9}{*}{ Asparagaceae } & Asparagus acutifolius L. & Mediterranean region & & 2 & \\
\hline & Asparagus horridus L. in J.A.Murray & Europe & & 12 & \\
\hline & Camassia sp. & California & 3,7 & & \\
\hline & Chlorogalum pomeridianum (DC.) Kunth & California & $2,3,5$ & & 9,11 \\
\hline & $\begin{array}{l}\text { Maianthemum racemosum (L.) Link } \\
\text { [Smilacina racemosa (L.) Link] }\end{array}$ & California & 7 & & \\
\hline & Ruscus aculeatus L. & Eurasia, Africa & & 1 & \\
\hline & Urginea maritima (L.) Baker & Eurasia, Africa & & 7 & \\
\hline & Yucca baccata Torrey & California & 3 & & \\
\hline & Yucca schidigera Roezl ex Ortgies & California, Mexico & & $1,3,4,7,8$ & \\
\hline
\end{tabular}


Table 3 Medicinal plants used before, during and after the Mission period, and present time at Mission Gardens. The numbers refer to emic and etic illness groupings (see Table 2) (Continued)

\begin{tabular}{|c|c|c|c|c|c|}
\hline Botanical family* & Medicinal plants* & Native & $\begin{array}{l}\text { Pre-Mission } \\
\text { period }\end{array}$ & Mission period & $\begin{array}{l}\text { Post- } \\
\text { Mission } \\
\text { period }\end{array}$ \\
\hline \multirow[t]{3}{*}{ Asphodelaceae } & Aloe sp. & California & & $1,3,4$ & \\
\hline & Aloe maculata All. & $\begin{array}{l}\text { Africa introduced from } \\
\text { Europe }\end{array}$ & & 3,7 & \\
\hline & Aloe vera (L.) Burm. fil. & Asia introduced from Europe & & 3 & \\
\hline \multirow[t]{2}{*}{ Aspleniaceae } & Asplenium trichomanes L. ssp. trichomanes & Eurasia, California & & 10,11 & \\
\hline & Ceterach officinarum Willd. & $\begin{array}{l}\text { Eurasia, Mediterranean } \\
\text { region }\end{array}$ & & 1,4 & \\
\hline \multirow[t]{34}{*}{ Asteraceae } & $\begin{array}{l}\text { Acamptopappus sphaerocephalus } \\
\text { (A. Gray) A. Gray }\end{array}$ & California & 11 & & \\
\hline & Achillea sp. & California & 9,11 & & \\
\hline & Achillea ageratum $\mathrm{L}$. & Europe & & 6,8 & \\
\hline & Achillea millefolium L. ssp. millefolium & California, Europe & $1,3,9,10,11$ & $\begin{array}{l}1,3,4,7,8,10 \\
11\end{array}$ & \\
\hline & $\begin{array}{l}\text { Acourtia microcephala DC. } \\
\text { [Perezia microcephala (DC) A. Gray] }\end{array}$ & California & $1,3,4,10,11$ & & \\
\hline & Ageratina sp. & California & & & 3,11 \\
\hline & $\begin{array}{l}\text { Ageratina adenophora (Spreng.) } \\
\text { R.M.King \& H.Rob. }\end{array}$ & Mexico & & & 3,11 \\
\hline & Ambrosia monogyra Torr. \& Gray & California & 3,11 & & \\
\hline & Ambrosia pilostachya DC. & California & 3,7 & & \\
\hline & $\begin{array}{l}\text { Amphipterygium adstringens } \\
\text { (Schltdl.) Schiede ex Standl. }\end{array}$ & Mexico & & $1,4,7$ & \\
\hline & Anacyclus clavatus Pers. & Europe & & 4,8 & \\
\hline & Anthemis arvensis L. ssp. arvensis & Eurasia, Africa & & $1,4,8,9$ & \\
\hline & Arctium minus Bernh. & Europe & & 3 & 7 \\
\hline & Artemisia sp. & California & $\begin{array}{l}4,5,7,9,10 \\
11\end{array}$ & & \\
\hline & Artemisia abrotanum $\mathrm{L}$. & Eurasia, Africa & & 3 & \\
\hline & Artemisia absinthium L. & Europe & & & 4 \\
\hline & Artemisia alba Turra & Europe & & 4 & \\
\hline & Artemisia californica Less. & California & $\begin{array}{l}3,5,7,8,9 \\
10,11\end{array}$ & & \\
\hline & $\begin{array}{l}\text { Artemisia cana Pursh ssp. } \\
\text { bolanderi (A. Gray) G. Ward }\end{array}$ & California & & & $1,3,8$ \\
\hline & $\begin{array}{l}\text { Artemisia douglasiana Besser } \\
\text { (A. heterophylla Nutt.) }\end{array}$ & California & $\begin{array}{l}1,3,4,5,6,7 \\
8,9,10,11\end{array}$ & & \\
\hline & Artemisia drancunuloides L. & California, Europe & $\begin{array}{l}1,4,6,7,8,9 \\
11\end{array}$ & & 8 \\
\hline & Artemisia herba-alba Asso & Europe & & 11 & \\
\hline & Artemisia mexicana Willd. & Mexico and South West USA & & 4,7 & \\
\hline & Artemisia ludoviciana Nutt. & California, Mexico & $3,8,10,11$ & $4,6,10,11$ & 4 \\
\hline & Artemisia pycnocephala DC & California & & & \\
\hline & Artemisia tridentate Nutt & California & $3,4,8,10,11$ & & \\
\hline & Baccharis glutinosa Pers. [B. salicifolia (Ruiz Lopez \& Pavon) Pers.] & California, Mexico & $3,9,11,13$ & $3,4,11$ & \\
\hline & Baccharis pilularis DC. & California & 3,11 & & \\
\hline & Baccharis plummerae A. Gray & California & 8,13 & & \\
\hline & Baccharis pteronioides A. Gray & Mexico, South West USA & & & $3,7,8,11$ \\
\hline & Baccharis sarothroides A. Gray & California & & 7,10 & \\
\hline & Balsamorhiza sagittalta (Pursh) Nutt. & California & $4,6,8,10,11$ & & $8,10,11$ \\
\hline & Bidens aurea (Aiton) Sherff & Mexico & & 4 & \\
\hline & Calea urticifolia (Mill.) DC. & Mexico & & 6,12 & \\
\hline
\end{tabular}


Table 3 Medicinal plants used before, during and after the Mission period, and present time at Mission Gardens. The numbers refer to emic and etic illness groupings (see Table 2) (Continued)

\begin{tabular}{|c|c|c|c|c|c|}
\hline Botanical family* & Medicinal plants* & Native & $\begin{array}{l}\text { Pre-Mission } \\
\text { period }\end{array}$ & Mission period & $\begin{array}{l}\text { Post- } \\
\text { Mission } \\
\text { period }\end{array}$ \\
\hline & $\begin{array}{l}\text { Calea zacatechichi Schltdl. } \\
\text { (C. ternifolia Kunth.) }\end{array}$ & Mexico & & 4,12 & \\
\hline & Calendula arvensis L. & Europe & & $3,8,10,11$ & \\
\hline & Calendula officinalis L. & $\begin{array}{l}\text { Europe, naturalized in } \\
\text { California }\end{array}$ & & 3 & \\
\hline & $\begin{array}{l}\text { Carduus pynocephalus L. ssp. } \\
\text { pynocephalus }\end{array}$ & Europe & & 3 & \\
\hline & $\begin{array}{l}\text { Carlina acanthifolia All. ssp. cynara } \\
\text { (Pourret ex Duby) Rouy }\end{array}$ & Europe & & 13 & \\
\hline & Centaurea aspera $\mathrm{L}$. & Europe & & 1,5 & \\
\hline & Chamaemelum nobile (L.) All. & $\begin{array}{l}\text { Europe, naturalized in } \\
\text { California }\end{array}$ & & $\begin{array}{l}2,3,4,8,9,10 \\
11,14\end{array}$ & \\
\hline & $\begin{array}{l}\text { Chamomilla recutita (L.) Rauschert } \\
\text { (Matricaria chamomilla L.) }\end{array}$ & $\begin{array}{l}\text { Europe, naturalized in } \\
\text { California }\end{array}$ & & 4 & \\
\hline & $\begin{array}{l}\text { Chamomilla suaveolens (Pursh) Rydb. } \\
\text { (Matricaria discoidea DC.) }\end{array}$ & $\begin{array}{l}\text { Europe, naturalized in } \\
\text { California }\end{array}$ & $\begin{array}{l}3,4,5,6,7 \\
10,11\end{array}$ & & \\
\hline & Chaptalia nutans (L.) Polak. & Caribbean & & 3,7 & \\
\hline & $\begin{array}{l}\text { Chrysanthemum balsamita } \mathrm{L} \text {. } \\
\text { (Tanacetum balsamita L.) }\end{array}$ & $\begin{array}{l}\text { Europe, naturalized in } \\
\text { California }\end{array}$ & & & \\
\hline & Cichorium intybus $\mathrm{L}$. & $\begin{array}{l}\text { Europe, naturalized in } \\
\text { California }\end{array}$ & & & 1,4 \\
\hline & Cirsium sp. & California & $3,4,10$ & & \\
\hline & Cirsium arvense (L.) Scop. & $\begin{array}{l}\text { Europe, naturalized in } \\
\text { California }\end{array}$ & & 3 & \\
\hline & $\begin{array}{l}\text { Conyza canadensis (L.) Cronq. } \\
\text { (Erigeron canadensis L.) }\end{array}$ & California & 8,12 & & \\
\hline & $\begin{array}{l}\text { Corethrogyne filaginifolia (Hook. } \\
\text { \& Arn.) Nutt. }\end{array}$ & California & $1,10,11$ & & \\
\hline & Cynara scolymus L. & Mediterranean region & & 4,5 & \\
\hline & $\begin{array}{l}\text { Deinandra fasciculate (DC.) Greene } \\
\text { [Hemizonia fasciculata (DC.) Torr. \& A. Gray] }\end{array}$ & California & 8 & & \\
\hline & Encelia californica Nutt. & California & & & \\
\hline & Encelia farinose Torrey \& A. Gray & California & 1,9 & & \\
\hline & Ericameria arborescens (A. Gray) E. Greene & California & $\begin{array}{l}1,3,4,5,7,9 \\
10,11\end{array}$ & & \\
\hline & Ericameria laricifolia (A. Gray) Shinn. & California & 11 & & \\
\hline & $\begin{array}{l}\text { Ericameria nauseosa (Pall. Ex Pursh) G. } \\
\text { L. Nesom \& Baird [Bigilovia nauseosa M. E. } \\
\text { Jones; Chrysothamnus nauseosa } \\
\text { (Pall. Ex Pursh) Britton] }\end{array}$ & California & $1,9,10$ & & \\
\hline & $\begin{array}{l}\text { Ericameria palmeri (A. Gray) H. M. Hall var. } \\
\text { pachylepsis (H. M. Hall) G. Nesom } \\
\text { [E. acradenius (Greene) S. F. Blake; } \\
\text { Haplopappus palmeri A. Gray; } \\
\text { Aplopappus palmeri Gray] }\end{array}$ & California & $3,10,11$ & & \\
\hline & $\begin{array}{l}\text { Erigeron canadensis L. [Conyza } \\
\text { canadensis (L.) Cronq.) }\end{array}$ & California & $4,8,13$ & & \\
\hline & $\begin{array}{l}\text { Erigeron foliosus Nutt. var. foliosus } \\
\text { (E. foliosus Nutt. var. stenophyllus; } \\
\text { E. utahensis Gray) }\end{array}$ & California & 11 & & \\
\hline & Erigeron karwinskianus DC. & Mexico & & $4,6,14$ & \\
\hline & Eriophyllum confertiflorum (DC.) A. Gray & California & 7 & & \\
\hline & Eupatorium perfoliatum L. & Eastern USA & & & 3,11 \\
\hline & Franseria ambrosioides (Cav.) Payne & California & & 3,7 & \\
\hline & Gnaphalium sp. & California & & 10,12 & \\
\hline & Gnaphalium bicolor Bioletti & California & 1,4 & & \\
\hline
\end{tabular}


Table 3 Medicinal plants used before, during and after the Mission period, and present time at Mission Gardens. The numbers refer to emic and etic illness groupings (see Table 2) (Continued)

\begin{tabular}{|c|c|c|c|c|c|}
\hline Botanical family* & Medicinal plants* & Native & $\begin{array}{l}\text { Pre-Mission } \\
\text { period }\end{array}$ & Mission period & $\begin{array}{l}\text { Post- } \\
\text { Mission } \\
\text { period }\end{array}$ \\
\hline & $\begin{array}{l}\text { [Pseudognaphalium bioletti } \\
\text { (Bioletti) A. Anderb.] }\end{array}$ & & & & \\
\hline & $\begin{array}{l}\text { Gnaphalium canescens DC. } \\
\text { [Pseudognaphalium canescens (DC.) } \\
\text { W.A. Weber] }\end{array}$ & California & $1,3,4,10,11$ & & \\
\hline & $\begin{array}{l}\text { Grindelia camporum E. Greene } \\
\text { (G. robusta Nutt.) }\end{array}$ & California & $1,3,10,11$ & & \\
\hline & Grindelia hirsutala Hook. \& Arn. & California & 10 & & \\
\hline & Grindelia stricta DC. (G. latifolia Kellogg) & California & 3 & & \\
\hline & Gutierrezia microcephala (DC.) A. Gray & California & 9 & & \\
\hline & Helenium mexicanum Kunth & Mexico & & 10 & \\
\hline & Helenium puberulum DC. & California & $3,6,10,11$ & & \\
\hline & Helianthus annuus L. & Europe & & 3,8 & \\
\hline & Helichrysum italicum G. Don f. & Mediterranean region & & 4 & \\
\hline & $\begin{array}{l}\text { Helichrysum stoechas (L.) Moench } \\
\text { spp. stoechas }\end{array}$ & Mediterranean region & & $1,4,8,11$ & \\
\hline & Heterotheca grandiflora Nutt. & California & 3,6 & & \\
\hline & Heterotheca inuloides Cass. & Mexico & & 3,10 & \\
\hline & Inula montana $\mathrm{L}$. & Western mediterranean & & 3 & \\
\hline & Inula viscosa (L.) Ait. & Mediterranean region & & 3 & \\
\hline & Jasonia glutinosa (L.) DC. & Europe, Africa & & $4,8,14$ & \\
\hline & Jasonia tuberosa (L.) DC. & Europe & & $3,4,7,11$ & \\
\hline & Leptosyne maritime (Nutt.) A. Gray & California & 4 & & \\
\hline & Madia sativa Molina & California & 7,10 & & 7 \\
\hline & Matricaria discoidea DC. & Asia, North West USA & & 4,8 & $4,6,7,10$ \\
\hline & Matricaria recutita L. & Europe & & & \\
\hline & Mikania sp. & Mexico and West USA & & 4,12 & \\
\hline & Montanoa tomentosa Cerv. & Mexico & & 7 & \\
\hline & Onopordum acanthium L. & Eurasia & & 3,4 & \\
\hline & Parthenium hysterophorus L. & Mexico & & & \\
\hline & Phagnalon saxatile (L.) Cass. & Mediterranean region & & 2 & \\
\hline & $\begin{array}{l}\text { Pleiacanthus spinosus (Nutt.) Rydb. } \\
\text { (Lygodesmia spinosa Nutt.) }\end{array}$ & California & 2 & & \\
\hline & Polymnia maculata Cav. & Mexico & & $3,4,6$ & \\
\hline & $\begin{array}{l}\text { Pseudognaphalium californicum (DC.) } \\
\text { Anderb. (Gnaphalium decurrens E. Ives) }\end{array}$ & California & $4,8,10,11$ & & \\
\hline & $\begin{array}{l}\text { Pseudognaphalium canescens (DC.) } \\
\text { W. A. Weber [Gnaphalium canescens DC.] }\end{array}$ & California & 7 & & \\
\hline & Santolina chamaecyparissus L. & Europe & & $3,4,8$ & \\
\hline & $\begin{array}{l}\text { Santolina chamaecyparissus L. ssp. } \\
\text { squarrosa (DC.) Nyman }\end{array}$ & Europe & & $1,4,8,9,11$ & \\
\hline & $\begin{array}{l}\text { Santolina chamaecyparissus } \\
\text { L. ssp. magonica O.Bolòs, R.Mol. et } \\
\text { P.Monts. var. teucrietorum O.Bolòs et Vigo }\end{array}$ & Europe & & $\begin{array}{l}3,4,7,8,9,10 \\
11,12\end{array}$ & \\
\hline & Senecio angulifolius DC. & Mexico & & 3,9 & \\
\hline & $\begin{array}{l}\text { Senecio flaccidus Less. var. douglasii } \\
\text { (DC.) B.L. Turner \& T.M. Barkley } \\
\text { (S. douglasii DC.) }\end{array}$ & California & $3,6,7,10,13$ & & \\
\hline & $\begin{array}{l}\text { Solidago californica Nutt. [S. velutina } \\
\text { DC. ssp. californica (Nutt.) Semple] }\end{array}$ & California & $\begin{array}{l}3,4,5,9,10 \\
11\end{array}$ & & \\
\hline & Sonchus asper (L.) Hill & Eurasia, Africa & & 3 & \\
\hline & Sonchus oleraceus L. & Eurasia & & 3 & \\
\hline
\end{tabular}


Table 3 Medicinal plants used before, during and after the Mission period, and present time at Mission Gardens. The numbers refer to emic and etic illness groupings (see Table 2) (Continued)

\begin{tabular}{|c|c|c|c|c|c|}
\hline Botanical family* & Medicinal plants* & Native & $\begin{array}{l}\text { Pre-Mission } \\
\text { period }\end{array}$ & Mission period & $\begin{array}{l}\text { Post- } \\
\text { Mission } \\
\text { period }\end{array}$ \\
\hline & Sonchus tenerrimus $L$. & Europe, Africa, Middle East & & 4 & \\
\hline & Tagetes erecta $\mathrm{L}$. & Mexico & & $4,6,9,10,12$ & \\
\hline & Tagetes lucida (Sweet) Voss & Mexico & & $3,4,7,12$ & \\
\hline & Tanacetum balsamita $\mathrm{L}$. & Europe & & 3,4 & \\
\hline & Tanacetum corymbosum (L.) Sch. Bip. & Europe & & 4 & \\
\hline & Tanacetum parthenium (L.) Sch. Bip. & Eurasia & & $4,8,13$ & \\
\hline & Tanacetum vulgare $\mathrm{L}$. & Europe & & 4,8 & 4 \\
\hline & Taraxacum officinale Weber & Europe & & $1,3,4,12$ & \\
\hline & $\begin{array}{l}\text { Thelesperma gracile (Torr.) A. Gray } \\
\text { [T. megapotamicum (Spreng.) Kuntze] }\end{array}$ & Mexico and South West USA & & 4,8 & \\
\hline & Tithonia diversifolia (Hemsl.) A. Gray & Mexico & & $3,6,9$ & \\
\hline & Trixis californica Kellogg & California and Mexico & & 3,9 & \\
\hline & Tussilago farfara $\mathrm{L}$. & Eurasia & & 3,11 & \\
\hline & Verbesina sp. & California & & & 3 \\
\hline & Wyethia angustifolia (DC.) Nutt. & California & 3,10 & & \\
\hline & Wyethia helenioides (DC.) Nutt. & California & $3,5,9,10$ & & \\
\hline & Xanthium strumarium L. & California & 3,13 & & \\
\hline Begoniaceae & Begonia heracleifolia Cham. \& Schltdl. & Mexico & & 3,4 & \\
\hline \multirow[t]{2}{*}{ Berberidaceae } & Berberis aquifolium Pursh & California & 11 & & \\
\hline & Berberis nevinii A. Gray & California & & & \\
\hline \multirow[t]{5}{*}{ Betulaceae } & Alnus sp. & California & 3,4 & & \\
\hline & Alnus arguta (Schltdl.) Spach & Mexico & & 3,12 & \\
\hline & Betula occidentalis Hook. & California & & & $4,10,11$ \\
\hline & Betula pendula Roth & Europe & & 2 & \\
\hline & $\begin{array}{l}\text { Corylus cornuta var. californica } \\
\text { (A. DC.) E. Murray }\end{array}$ & California & & & 11 \\
\hline \multirow[t]{3}{*}{ Bignoniaceae } & Crescentia cujete L. & Mexico & & 3,4 & \\
\hline & Parmentiera edulis DC. & Mexico & & $9,10,12$ & \\
\hline & Tabebuia rosea (Bertol.) DC. & Mexico & & $3,7,12$ & \\
\hline \multirow[t]{2}{*}{ Bixaceae } & Bixa orellana $\mathrm{L}$. & Mexico & & 3,6 & \\
\hline & $\begin{array}{l}\text { Cochlospermum vitifolium } \\
\text { (Willd.) Spreng. }\end{array}$ & Mexico & & 3,4 & \\
\hline \multirow[t]{12}{*}{ Boraginaceae } & Borago officinalis $\mathrm{L}$. & $\begin{array}{l}\text { Europe, naturalized in } \\
\text { California }\end{array}$ & & $1,2,3.6,11$ & $1,4,10$ \\
\hline & $\begin{array}{l}\text { Cordia curassavica (Jacq.) } \\
\text { Roem. \& Schult. }\end{array}$ & Mexico & & $3,5,8,10$ & \\
\hline & Ehretia tinifolia L. & Mexico & & 12 & \\
\hline & $\begin{array}{l}\text { Eriodictyon californicum } \\
\text { (Hook. \& Arn.) Torrey }\end{array}$ & California & $\begin{array}{l}1,3,4,6,7,8 \\
10,11\end{array}$ & & $8,9,10$ \\
\hline & Eriodictyon crassifolium Benth. & California & $1,3,7,10,11$ & & \\
\hline & Eriodictyon trichocalyx A. Heller & California & $\begin{array}{l}1,3,6,7,10 \\
11\end{array}$ & & \\
\hline & $\begin{array}{l}\text { Heliotropium curvassavicum } \\
\text { L. var. oculatum }\end{array}$ & California & 5 & & \\
\hline & Lithospermum officinale L. & Europe & & 4 & \\
\hline & Phacelia distans Benth. & California & & & $1,3,4,10$ \\
\hline & Phacelia ramoisissima Lehm. & California & $6,10,11$ & & \\
\hline & Pulmonaria longifolia (Bast.) Boreau & Europe & & 11 & \\
\hline & Symphytum asperum Lepech. & $\begin{array}{l}\text { Asia, introduced from } \\
\text { Europe }\end{array}$ & & & $1,8,10$ \\
\hline
\end{tabular}


Table 3 Medicinal plants used before, during and after the Mission period, and present time at Mission Gardens. The numbers refer to emic and etic illness groupings (see Table 2) (Continued)

\begin{tabular}{|c|c|c|c|c|c|}
\hline Botanical family* & Medicinal plants* & Native & $\begin{array}{l}\text { Pre-Mission } \\
\text { period }\end{array}$ & Mission period & $\begin{array}{l}\text { Post- } \\
\text { Mission } \\
\text { period }\end{array}$ \\
\hline & Symphytum officinale L. & Europe & & 7 & \\
\hline & Symphytum tuberosum L. ssp. tuberosum & Europe & & 7 & \\
\hline & Tournefortia hartwegiana DC. & Mexico & & $4,7,10,13$ & \\
\hline \multirow[t]{12}{*}{ Brassicaceae } & Brassica nigra (L.) Koch & Eurasia, Africa & & 11 & \\
\hline & Brassica oleracea L. ssp. oleracea & Europe & & $3,4,8$ & \\
\hline & Brassica rapa L. & Europe, Asia & & & 7,10 \\
\hline & Capsella bursa-pastoris (L.) Medik. & Eurasia & & $1,4,5,10$ & \\
\hline & Coronopus didymus (L.) Sm. & South America & & $1,6,11$ & \\
\hline & Coronopus squamatus (Forsk.) Asch. & Mediterranean region & & 1,2 & \\
\hline & $\begin{array}{l}\text { Descurainia pinnata (Walter) Britton } \\
\text { [Sisymbrium canescens (Phil.) Reiche., } \\
\text { S. pinnatum (Walter) Britton] }\end{array}$ & California & 4 & & \\
\hline & Lepidium Iatifolium L. & Eurasia & & 12 & \\
\hline & Lepidium nitidum Torrey \& Gray & California & 4,6 & & \\
\hline & $\begin{array}{l}\text { Raphanus raphanistrum L. ssp. sativus } \\
\text { (L.) Domin }\end{array}$ & Europe & & & 3 \\
\hline & Rorippa sp. & California & 11 & & \\
\hline & $\begin{array}{l}\text { Rorippa nasturtium-aquaticum (L.) } \\
\text { Hayek (Nasturtium officinalis W. T. Aiton) }\end{array}$ & $\begin{array}{l}\text { Europe, Asia, naturalized in } \\
\text { California and Mexico }\end{array}$ & 1,4 & $1,6,12,14$ & \\
\hline \multirow[t]{4}{*}{ Burseraceae } & Bursera grandifolia (Schltdl.) Engl. & Mexico & & $4,8,12$ & \\
\hline & Bursera microphylla A. Gray & California & 3 & & \\
\hline & Bursera simaruba (L.) Sarg. & Mexico & & 3,12 & \\
\hline & Protium copal Engl. & Mexico & & $1,3,4,7$ & \\
\hline \multirow[t]{2}{*}{ Buxaceae } & Buxus balearica Lam. & Eurasia, Africa & & 4 & \\
\hline & Buxus sempervirens $\mathrm{L}$. & Europe & & 4 & \\
\hline \multirow[t]{8}{*}{ Cactaceae } & $\begin{array}{l}\text { Cylindropuntia acanthrocarpa } \\
\text { (Engelm. \& Bigelow) F. M. Knuth } \\
\text { (Opuntia acanthrocarpa } \\
\text { Engelm. \& Bigelow) }\end{array}$ & California & 3 & & \\
\hline & $\begin{array}{l}\text { Lemaireocereus thurberi (Engelm.) } \\
\text { Britton \& Rose [Stenocereus thurberi } \\
\text { (Engelm.) Britton \& Rose] }\end{array}$ & Mexico & & $1,3,12$ & \\
\hline & Lophophora williamsii (Lem.) J. M. Coult. & Mexico, Texas & & 3 & 1,4 \\
\hline & Opuntia maxima A.Berger & $\begin{array}{l}\text { Mexico introduced from } \\
\text { Europe }\end{array}$ & & $\begin{array}{l}1,2,3,4,5,11 \\
12\end{array}$ & \\
\hline & Opuntia sp. & California and Mexico & $1,3,7$ & 1,4 & 10 \\
\hline & $\begin{array}{l}\text { Opuntia imbricate DC. [Cylindropuntia } \\
\text { imbricate (DC.) Haw.] }\end{array}$ & Mexico & & 4,10 & \\
\hline & Opuntia leucotricha DC. & Mexico & & $1,4,12$ & \\
\hline & $\begin{array}{l}\text { Opuntia tuberosus (Pfeiff.) } \\
\text { Britton \& Rose }\end{array}$ & California and Mexico & & 8 & \\
\hline \multirow[t]{2}{*}{ Cannabaceae } & Cannabis sativa $\mathrm{L}$. & Eastern Asia & & & \\
\hline & Humulus lupulus L. & Europe & & 8 & \\
\hline \multirow[t]{6}{*}{ Caprifoliaceae } & Lonicera sp. & California & 7,10 & & \\
\hline & Lonicera implexa Ait. & Europe & & $1,3,5$ & \\
\hline & Lonicera interrupta Benth. & California & $3,9,10$ & & \\
\hline & $\begin{array}{l}\text { Lonicera subspicata Hook. \& } \\
\text { Arn.var. subspicata }\end{array}$ & California & 3,10 & & \\
\hline & Scabiosa sp. & Europe & & & 1 \\
\hline & Valeriana officinalis $\mathrm{L}$. & Europe & & & \\
\hline Caricaceae & Carica papaya L. & Europe & & $3,4,10$ & \\
\hline
\end{tabular}


Table 3 Medicinal plants used before, during and after the Mission period, and present time at Mission Gardens. The numbers refer to emic and etic illness groupings (see Table 2) (Continued)

\begin{tabular}{|c|c|c|c|c|c|}
\hline Botanical family* & Medicinal plants* & Native & $\begin{array}{l}\text { Pre-Mission } \\
\text { period }\end{array}$ & Mission period & $\begin{array}{l}\text { Post- } \\
\text { Mission } \\
\text { period }\end{array}$ \\
\hline \multirow[t]{5}{*}{ Caryophyllaceae } & $\begin{array}{l}\text { Herniaria hirsuta L. ssp. cinerea } \\
\text { (DC. in Lam. et DC.) Arcang. }\end{array}$ & Eurasia, Africa & & $2,8,12$ & \\
\hline & Paronychia argentea Lam. & Mediterranean region & & 1 & \\
\hline & $\begin{array}{l}\text { Silene laciniata Cav. ssp. major C. } \\
\text { Hitchc. \& Maguire (S. laciniata } \\
\text { Cav. ssp. laciniata) }\end{array}$ & California & 5,7 & & \\
\hline & $\begin{array}{l}\text { Spergularia salina J. Presl \& C. } \\
\text { Presl [S. marina (L.) Besser] }\end{array}$ & California & & & $\begin{array}{l}1,3,4,9 \\
10,11\end{array}$ \\
\hline & Stellaria media (L.) Vill. & Europe & & & \\
\hline \multirow[t]{2}{*}{ Celastraceae } & Hippocratea excelsa Kunth & Mexico & & 3 & \\
\hline & $\begin{array}{l}\text { Torreya californica Torrey } \\
\text { [Tumion californicum } \\
\text { (Torrey) Greene] }\end{array}$ & California & $4,8,11$ & & \\
\hline \multirow[t]{2}{*}{ Cistaceae } & Cistus albidus L. & Europe. Africa & & $3,8,11$ & \\
\hline & Cistus salviifolius L. & Eurasia, Africa & & 3,12 & \\
\hline \multirow[t]{2}{*}{ Commelinaceae } & Commrlina erecta $\mathrm{L}$. & Mexico & & $4,7,12$ & \\
\hline & $\begin{array}{l}\text { Rhoeo discolor (L'Hér.) Hance } \\
\text { (Tradescantia spathacea Sw.) }\end{array}$ & Mexico & & $3,6,7$ & \\
\hline \multirow[t]{4}{*}{ Convolvulaceae } & Cuscuta sp. & California and Mexico & & 4,12 & \\
\hline & Cuscuta californica Hook. \& Arn. & California & 11 & & \\
\hline & $\begin{array}{l}\text { Ipomoea arborescens } \\
\text { (Humb. \& Bonpl. Ex. Willd.) G. Don }\end{array}$ & Mexico & & $1,4,8,12$ & \\
\hline & Ipomoea stans Cav. & Mexico & & 4,8 & \\
\hline Cornaceae & $\begin{array}{l}\text { Cornus sericea L. ssp. californica } \\
\text { (C. californica C.AQ. Meyer) }\end{array}$ & California & 11 & & \\
\hline \multirow[t]{9}{*}{ Crassulaceae } & $\begin{array}{l}\text { Dudleya pulverulenta (Nutt.) } \\
\text { Britton \& Rose }\end{array}$ & California & 3,10 & & \\
\hline & $\begin{array}{l}\text { Hylotelephium maximum } \\
\text { (L.) Holub }\end{array}$ & Eurasia & & 3,8 & \\
\hline & $\begin{array}{l}\text { Hylotelephium telephium } \\
\text { (L.) H. Ohba }\end{array}$ & Eurasia & & 3 & \\
\hline & Kalanchoe pinnata (Lam.) Pers. & Madagascar & & 3 & \\
\hline & Sedum oxypetalum Kunth & Mexico & & 3 & \\
\hline & Sedum spathulifolium Hook. & California & $3,10,11$ & & \\
\hline & Sedum spurium M. Bieb. & Asia introduced from Europe & & 3 & \\
\hline & Sempervivum tectorum $\mathrm{L}$. & Europe & & 9 & \\
\hline & Umbilicus rupestris (Salisb.) Dandy & Europe & & 3 & \\
\hline \multirow[t]{11}{*}{ Cucurbitaceae } & $\begin{array}{l}\text { Citrullus lanatus var. lanatus } \\
\text { (Thunb.) Matsum. \& Nakai }\end{array}$ & Africa & & & 6,11 \\
\hline & Cucumis sativus L. & $\begin{array}{l}\text { Asia, introduced from } \\
\text { Europe }\end{array}$ & & 4 & \\
\hline & Cucurbita foetidissima Kunth & California and Mexico & $3,4,7,11$ & 12 & 3,7 \\
\hline & Cucurbita maxima Duchesne. & South America & & 4 & \\
\hline & Cucurbita palmate S. Wats. & California & & & 4,11 \\
\hline & Cucurbita pepo L. & South America & & 12 & \\
\hline & Ibervillea sonorae S. Wats. & Mexico & & 6 & \\
\hline & Luffa aegyptiaca Mill. & $\begin{array}{l}\text { Egypt, introduced from } \\
\text { Europe }\end{array}$ & & 3 & \\
\hline & Marah fabacea (Naudin) Greene & California & 3,13 & & 3 \\
\hline & Marah macrocarpus E. Greene & California & $\begin{array}{l}1,3,4,5,7,9 \\
11\end{array}$ & & \\
\hline & Momordica charantia L. & South Indian & & $4,8,12$ & \\
\hline Cupressaceae & Hesperocyparis macrocarpa & California & 7 & & \\
\hline
\end{tabular}


Table 3 Medicinal plants used before, during and after the Mission period, and present time at Mission Gardens. The numbers refer to emic and etic illness groupings (see Table 2) (Continued)

\begin{tabular}{|c|c|c|c|c|c|}
\hline Botanical family* & Medicinal plants* & Native & $\begin{array}{l}\text { Pre-Mission } \\
\text { period }\end{array}$ & Mission period & $\begin{array}{l}\text { Post- } \\
\text { Mission } \\
\text { period }\end{array}$ \\
\hline & $\begin{array}{l}\text { (Hartw.) Bartel (Cupressus } \\
\text { macrocarpa Hartw.) }\end{array}$ & & & & \\
\hline & Juniperus californica Carr. & California & $7,11,13$ & & \\
\hline & Juniperus chinensis $\mathrm{L}$. & Asia & & & \\
\hline & Juniperus communis $\mathrm{L}$. & Eurasia & & $3,4,7,9$ & \\
\hline & Juniperus deppeana Steud. & Mexico and South West USA & & 7,12 & \\
\hline & Juniperus phoenicea L. & Mediterranean region & & 3 & \\
\hline & Sequoia sempervirens (D. Don) Endl. & California & $3,9,10,11$ & & \\
\hline & Taxodium mucronatum Ten. & Mexico and South West USA & & $3,6,9$ & \\
\hline \multirow[t]{4}{*}{ Cyperaceae } & Schoenoplectus sp. & California & 3,7 & & \\
\hline & Scirpus sp. & California & 7 & & \\
\hline & $\begin{array}{l}\text { Scirpus acutus L. var. occidentalis } \\
\text { (S. Watson) Beetle [Schoenoplectus } \\
\text { acutus (Muhl. Ex Bigelow) A. } \\
\text { Love \& D. Love var. occidentalis } \\
\text { (S. Watson) S. G. Sm.] }\end{array}$ & California & 3,7 & & \\
\hline & $\begin{array}{l}\text { Scirpus californicus (C. Mewyer) } \\
\text { Steudel (Schoenoplectus } \\
\text { californicus C. A. Mey. Palla) }\end{array}$ & California & 3 & & \\
\hline Datiscaceae & Datisca glomerata (C. Presl) Baillon & California & $3,7,8,10$ & & \\
\hline Dennstaedtiaceae & Pteridium aquilinum (L.) Kuhn. & California, Europe, Mexico & & 1 & 11,13 \\
\hline \multirow[t]{2}{*}{ Dioscoreaceae } & Dioscorea sp. & Mexico & & $5,7,8$ & \\
\hline & Tamus communis $\mathrm{L}$. & Europe & & 3,7 & \\
\hline \multirow[t]{2}{*}{ Dryopteridaceae } & $\begin{array}{l}\text { Dryopteris arguta (Kaulf.) Watt } \\
\text { [Aspidium rigidum Sw. arguta (DC.) Eat.] }\end{array}$ & California & 3,11 & & \\
\hline & Dryopteris filix-mas (L.) Schott & Europe, California & & 4 & \\
\hline Ebenaceae & Diospyros kaki L.f. & $\begin{array}{l}\text { Asia introduced from } \\
\text { Europe, California }\end{array}$ & & 1,4 & \\
\hline \multirow[t]{3}{*}{ Ephedraceae } & Ephedra sp. & California & 6 & & \\
\hline & Ephedra californica S. Wats. & California & $\begin{array}{l}1,3,4,6,10 \\
11,13\end{array}$ & & \\
\hline & Ephedra viridis S. Watson & California & $\begin{array}{l}1,3,4,6,11 \\
13\end{array}$ & & \\
\hline \multirow[t]{6}{*}{ Equisetaceae } & Equisetum sp. & California & $4,10,11,13$ & & \\
\hline & Equisetum arvense $\mathrm{L}$. & California, Europe, Mexico & 3 & $1,2,3,7,11,12$ & 1,11 \\
\hline & Equisetum hyemale L. & Europe, Mexico & & & $1,11,12$ \\
\hline & $\begin{array}{l}\text { Equisetum laevigatum A. Braun } \\
\text { (E. funstoni A. A. Eaton) }\end{array}$ & California & $3,5,7,11,13$ & & \\
\hline & Equisetum ramossissimum Desf. & Eurasia, Africa & & 1,2 & \\
\hline & Equisetum telmateia Ehrh. & Eurasia, Africa & & $1,3,7,12$ & \\
\hline \multirow[t]{7}{*}{ Ericaceae } & Arbutus unedo L. & Europe & & 1 & \\
\hline & Arbutus menziesii Pursh & California & $3,4,10$ & & \\
\hline & Arbutus xalapensis Kunth & Mexico, South West USA & & 7 & \\
\hline & Arctostaphylos glauca Lindl. & California & $3,4,13$ & 2,12 & \\
\hline & Arctostaphylos uva-ursi (L.) Spreng. & California and Europe & & 12 & 3 \\
\hline & Erica cinerea $\mathrm{L}$. & Europe & & 12 & \\
\hline & Vaccinium sp. & California & 12 & & \\
\hline \multirow[t]{3}{*}{ Euphorbiaceae } & Acalypha alopecuroidea Jacq. & Mexico & & 4 & \\
\hline & Chamaesyce sp. (Euphorbia sp.) & California & $3,9,11$ & & \\
\hline & Cnidoscolus chayamansa & Mexico & & $3,13,12$ & \\
\hline
\end{tabular}


Table 3 Medicinal plants used before, during and after the Mission period, and present time at Mission Gardens. The numbers refer to emic and etic illness groupings (see Table 2) (Continued)

\begin{tabular}{|c|c|c|c|c|c|}
\hline Botanical family* & Medicinal plants* & Native & $\begin{array}{l}\text { Pre-Mission } \\
\text { period }\end{array}$ & Mission period & $\begin{array}{l}\text { Post- } \\
\text { Mission } \\
\text { period }\end{array}$ \\
\hline & $\begin{array}{l}\text { Cnidoscolus urens L. ssp. } \\
\text { stimulosus (Michx.) Govaerts }\end{array}$ & Mexico & & & 11 \\
\hline & Croton sp. & Asia & & 4 & \\
\hline & Croton californicus Muell & California & $7,9,10$ & & \\
\hline & Croton draco Schldtl. & Mexico & & 9 & \\
\hline & Croton fragilis Schltr. & Mexico & & 4,6 & \\
\hline & $\begin{array}{l}\text { Croton setiger Hook. [Eremocarpus } \\
\text { setiger (Hook.) Benth.] }\end{array}$ & California & $4,6,8,11$ & & \\
\hline & $\begin{array}{l}\text { Eremocarpus setigerus } \\
\text { (Hook.) Benth. }\end{array}$ & California & & & $1,10,11$ \\
\hline & Euphorbia sp. & California & $3,6,9,11$ & 3 & \\
\hline & $\begin{array}{l}\text { Euphorbia albomarginata } \\
\text { Torrey \& A. Gray }\end{array}$ & California & & & \\
\hline & $\begin{array}{l}\text { Euphorbia amygdaloides L. ssp. } \\
\text { amygdaloides }\end{array}$ & Europa & & 3 & \\
\hline & Euphorbia antisyphillitica Zucc. & Mexico and South West USA & & $4,6,8,9$ & \\
\hline & $\begin{array}{l}\text { Euphorbia characias L. ssp. } \\
\text { characias }\end{array}$ & Europe & & 3 & \\
\hline & Euphorbia grantii Oliv. & Mexico, South West USA & & & 3 \\
\hline & Euphorbia lathyris L. & Eurasia, Africa & & 4 & \\
\hline & $\begin{array}{l}\text { Euphorbia ocellata Durand \& } \\
\text { Hilg. ssp. ocellata }\end{array}$ & California & & & \\
\hline & Euphorbia peplus L. & Eurasia, Africa & & 3 & \\
\hline & Euphorbia polycarpa Benth. & California & 11 & & 11 \\
\hline & Euphorbia serrata L. & Europe, Africa & & 3 & \\
\hline & $\begin{array}{l}\text { Euphorbia villosa Waldst. \& } \\
\text { Kit. ex Willd. }\end{array}$ & Europe & & 3 & \\
\hline & Jatropha cinerea (Oretga) Mull. & Mexico and South West USA & & 3,9 & \\
\hline & Jatropha curcas L. & Mexico & & $4,7,9$ & \\
\hline & Jatropha dioica Sesse & Mexico and Texas & & 3,9 & \\
\hline & Ricinus communis $\mathrm{L}$. & $\begin{array}{l}\text { Africa introduced from } \\
\text { Europe }\end{array}$ & & $4,8,12$ & \\
\hline & Synadenium grantii Hook. & Asia & & & 3 \\
\hline \multirow[t]{13}{*}{ Fabaceae } & Acacia cochliacantha Bonpl. ex Willd. & Mexico & & $4,6,10,12$ & \\
\hline & Acacia cornigera (L.) Willd. & Mexico & & 3 & \\
\hline & $\begin{array}{l}\text { Acacia farnesiana (L.) Willd. } \\
\text { [Vachellia farnesiana (L.) } \\
\text { Wight \& Arn.] }\end{array}$ & Mexico & & $1,4,6,11$ & \\
\hline & $\begin{array}{l}\text { Acmispon glaber (Vogel) Brouillet } \\
\text { [Lotus scoparius (Nutt. in Torr. } \\
\text { \& A. Gray) Ottley] }\end{array}$ & California & 10 & & \\
\hline & $\begin{array}{l}\text { Acosmium panamense (Benth.) } \\
\text { Yakoviev }\end{array}$ & Mexico & & $4,10,12$ & \\
\hline & Bauhinia divaricata $\mathrm{L}$. & Jamaica & & $4,6,10,12$ & \\
\hline & Caesalpinia pulcherrima (L.) Sw. & Mexico & & 10 & \\
\hline & Calliandra californica Benth. & California and Mexico & & 12 & \\
\hline & Ceratonia siliqua L. & Mediterranean region & & $3,4,11$ & \\
\hline & Cercis occidentalis Torrey & California & & & \\
\hline & Crotalaria incana L. & Mexico & & 10 & \\
\hline & Desmodium incanum DC. & Mexico & & $3,4,6,10,12$ & \\
\hline & Enterolobium cyclocarpum & Mexico & & 3 & \\
\hline
\end{tabular}


Table 3 Medicinal plants used before, during and after the Mission period, and present time at Mission Gardens. The numbers refer to emic and etic illness groupings (see Table 2) (Continued)

\begin{tabular}{|c|c|c|c|c|c|}
\hline Botanical family* & Medicinal plants* & Native & $\begin{array}{l}\text { Pre-Mission } \\
\text { period }\end{array}$ & Mission period & $\begin{array}{l}\text { Post- } \\
\text { Mission } \\
\text { period }\end{array}$ \\
\hline & Erythrina corallodendron L. & Mexico & & & 11 \\
\hline & $\begin{array}{l}\text { Eysenhardtia polystachya } \\
\text { (Ortega) Sarg. }\end{array}$ & Mexico & & 13 & \\
\hline & $\begin{array}{l}\text { Gliricidia sepium (Jacq.) } \\
\text { Kunth ex Walp }\end{array}$ & Mexico & & 6,12 & \\
\hline & Glycyrrhiza glabra L. & Eurasia & & 4 & \\
\hline & Haematoxylon brasiletto H. Karst & Mexico & & $8,12,13$ & \\
\hline & Haematoxylon campechianum L. & Mexico & & $1,4,6$ & \\
\hline & Hoita macrostachya (DC.) Rydb. & California & 3,11 & & \\
\hline & Hoita orbicularis (Lindl.) Rydb. & California & 1,11 & & \\
\hline & Indigofera suffruticosa Mill. & Mexico & & $3,4,6,11$ & \\
\hline & Inga jinicuil G. Don & Mexico & & $1,4,6$ & \\
\hline & Lathyrus vestitus Nutt. & California & 4,11 & & \\
\hline & Lens culinaris Medic. & Asia & & 14 & \\
\hline & Lupinus sp. & California & 11,13 & & \\
\hline & Lupinus arboreus Sims & California & & & \\
\hline & $\begin{array}{l}\text { Lupinus cytisoides J. Agardt } \\
\text { (L. latifolia J. Agardt.) }\end{array}$ & California & & & \\
\hline & Lysiloma acapulcensis Benth. & Mexico & & 3 & \\
\hline & Medicago sativa $\mathrm{L}$. & Asia introduced from Europe & & 1,8 & \\
\hline & Mimosa tenuiflora (Willd.) Poir. & Mexico & & 3,4 & \\
\hline & Mucuna pruriens (L.) DC. & Africa & & 4 & \\
\hline & Ononis spinosa $\mathrm{L}$. & Eurasia, Africa & & 4 & \\
\hline & Olneya tesota A. Gray & California and Mexico & & $4,10,12$ & \\
\hline & Phaseolus vulgaris L. & $\begin{array}{l}\text { Central America cultivated } \\
\text { from all continents }\end{array}$ & & 5 & \\
\hline & Pisum sativum L. & Mediterranean region & & 14 & \\
\hline & Pithecellobium dulce (Roxb.) Benth. & Mexico & & 4,10 & \\
\hline & Prosopis sp. & California and Mexico & & $3,4,9$ & \\
\hline & Prosopis juliflora (Sw.) DC. & Mexico & & $3,4,9$ & \\
\hline & Prosopis grandulosa Torr. & California & 3,9 & & \\
\hline & Stylosanthes viscosa (L.) Sw. & Mexico & & & 4,6 \\
\hline & Tamarindus indica $\mathrm{L}$. & India & & & \\
\hline & Trifolium sp. & California & 4 & & \\
\hline & Vicia faba $\mathrm{L}$. & Eurasia & & 4,10 & \\
\hline & Vicia gigantean Hook. & California & 4 & & \\
\hline & Castanea sativa Mill. & Eurasia & & 4 & \\
\hline & $\begin{array}{l}\text { Notholithocarpus densiflorus } \\
\text { (Hook. \& Arn.) Manos, C. H. } \\
\text { Cannon, \& S. Oh [Lithocarpus } \\
\text { densiflorus (Hook. \& Arn.) Rehd.] }\end{array}$ & California & 3,11 & & \\
\hline & Quercus sp. & California, Mexico & $1,3,9$ & $1,9,11$ & \\
\hline & Quercus agrifolia Nee & California & $1,3,4$ & & $3,4,10$ \\
\hline & Quercus dumosa Nutt. & California & $3,9,11$ & & \\
\hline & Quercus faginea Lam. & Mediterranean region & & 3 & \\
\hline & Quercus ilex L. & Europe & & $1,3,4,14$ & $3,4,10$ \\
\hline & Quercus ilex ssp. ballota (Desf.) Samp. & Mediterranean region & & 2,3 & \\
\hline & Quercus oleoides Schltdl. \& Cham. & Mexico & & 9 & \\
\hline & Quercus lobata Nee & California & 4 & & \\
\hline
\end{tabular}


Table 3 Medicinal plants used before, during and after the Mission period, and present time at Mission Gardens. The numbers refer to emic and etic illness groupings (see Table 2) (Continued)

\begin{tabular}{|c|c|c|c|c|c|}
\hline Botanical family* & Medicinal plants* & Native & $\begin{array}{l}\text { Pre-Mission } \\
\text { period }\end{array}$ & Mission period & $\begin{array}{l}\text { Post- } \\
\text { Mission } \\
\text { period }\end{array}$ \\
\hline & Quercus robur L. & Eurasia & & 4 & \\
\hline & Quercus turbinella Greene & California & 3,9 & & \\
\hline Frankeniaceae & $\begin{array}{l}\text { Frankenia salina (Molina) I. M. } \\
\text { Johnst. (F. grandifolia Cham. \& } \\
\text { Schltdl.) }\end{array}$ & California & 4 & & \\
\hline \multirow[t]{3}{*}{ Gentianaceae } & Centaurium enthraea Raf. & Europe & & $1,4,8,11$ & $1,10,11$ \\
\hline & $\begin{array}{l}\text { Centaurium venustum (A. Gray) } \\
\text { B. L. Rob. [Zeltnera venusta (Gray) } \\
\text { G.Mans.] }\end{array}$ & California & 1,11 & & 1,11 \\
\hline & $\begin{array}{l}\text { Zeltnera venusta (A. Gray) Mansion } \\
\text { (Erythraea venusta A. Gray) }\end{array}$ & California & $1,6,11$ & & \\
\hline \multirow[t]{3}{*}{ Geraniaceae } & Geranium lucidum L. & Eurasia, Africa & & 3 & \\
\hline & Geranium robertianum L. & Europe & & 4 & \\
\hline & Pelargonium sp. & $\begin{array}{l}\text { South Africa, Introduced } \\
\text { from Europe }\end{array}$ & & 4 & \\
\hline Gesneriaceae & $\begin{array}{l}\text { Konleria deppeana } \\
\text { (Schltdl. \& Cham.) Fritsch }\end{array}$ & Mexico & & 4,13 & \\
\hline Grossulariaceae & Ribes indecorum Eastw. & California & 9 & & \\
\hline \multirow[t]{3}{*}{ Hypericaceae } & Hypericum androsaemum L. & Eurasia & & 3 & \\
\hline & Hypericum balearicum $\mathrm{L}$. & Spain's Balearic Islands & & 5 & \\
\hline & Hypericum perforatum L. & Eurasia & & $3,4,7,8,12$ & \\
\hline Illiciaceae & Illicium verum Hook.f. & Asia & & 4 & \\
\hline \multirow[t]{4}{*}{ Iridaceae } & Crocus sativus L. & Europe & & & 8 \\
\hline & Iris sp. & California & 4 & & \\
\hline & Iris douglasiana Herbert & California & & & \\
\hline & Sisyrinchum bellum S. Watson & California & $4,5,7,11$ & & 10,11 \\
\hline \multirow[t]{2}{*}{ Juglandaceae } & Juglans californica S. Wats. & California & 1 & & \\
\hline & Juglans regia $\mathrm{L}$. & Balkan Peninsula, Asia & & $\begin{array}{l}1,2,3,4,5,6,7 \\
10,11\end{array}$ & \\
\hline \multirow[t]{2}{*}{ Juncaceae } & $\begin{array}{l}\text { Juncus sp. (mainly, J. effusus } L . ; \\
\text { inflexus } L ., \text { and } J \text {. conglomeratus L.) }\end{array}$ & Eurasia, Africa & & 3 & \\
\hline & Juncus textilis Buchenau & California & 3 & & \\
\hline Krameriaceae & $\begin{array}{l}\text { Krameria grayi Rose \& Painter } \\
\text { (K. bicolor S. Watson) }\end{array}$ & California & & $3,4,12$ & \\
\hline \multirow[t]{12}{*}{ Lamiaceae } & $\begin{array}{l}\text { Agastache mexicana (Kunth) } \\
\text { Lint \& Epling }\end{array}$ & Mexico & & 8 & \\
\hline & Calamintha nepeta (L.) Savi & Europe, Africa & & 8 & \\
\hline & $\begin{array}{l}\text { Clinopodium douglasii (Benth.) } \\
\text { Kuntze [Micromeria douglasii } \\
\text { (Benth.) Kuntze; Satureja douglasii } \\
\text { (Benth.) Briq.] }\end{array}$ & California & $\begin{array}{l}3,4,5,8,9 \\
10,11\end{array}$ & & 4 \\
\hline & Dracocephalum moldavica $\mathrm{L}$. & $\begin{array}{l}\text { Asia, introduced from } \\
\text { Europe }\end{array}$ & & 1 & \\
\hline & Hyptis mutabilis (Rich.) Briq. & Mexico & & 4 & \\
\hline & Hyptis stellulata Benth. & Mexico & & $3,4,7,8,9$ & \\
\hline & Hyptis verticillata Jacq. & Mexico & & 3,4 & \\
\hline & $\begin{array}{l}\text { Hyptis emoryi Torrey [Condea emoryi } \\
\text { (Torr.) Harely \& J. F. B. Pastore] }\end{array}$ & California & 1 & & 1 \\
\hline & $\begin{array}{l}\text { Lavandula angustifolia Mill. } \\
\text { (L. vera DC:; L. spica L.) }\end{array}$ & Europe & & & \\
\hline & Lavandula latifolia Medik. & Mediterranean region & & $3,8,14$ & \\
\hline & Lavandula spica $\mathrm{L}$. & Mediterranean region & & & 4 \\
\hline & Leonurus japonicus Hoult & Asia & & $5,6,10$ & \\
\hline
\end{tabular}


Table 3 Medicinal plants used before, during and after the Mission period, and present time at Mission Gardens. The numbers refer to emic and etic illness groupings (see Table 2) (Continued)

\begin{tabular}{|c|c|c|c|c|c|}
\hline Botanical family* & Medicinal plants* & Native & $\begin{array}{l}\text { Pre-Mission } \\
\text { period }\end{array}$ & Mission period & $\begin{array}{l}\text { Post- } \\
\text { Mission } \\
\text { period }\end{array}$ \\
\hline & Lepechinia calycina (Benth.) Epling & California & & & $7,10,11$ \\
\hline & Lepechinia caulescens (Ortega) Epling & Mexico & & 4,6 & \\
\hline & Marrubium vulgare L. & Europe & & $3,4,11$ & $3,6,8,10$ \\
\hline & Melissa officinalis L. & Europe & & $1,4,8,11$ & 1 \\
\hline & Mentha sp. & California & $3,4,7,8,9,13$ & & \\
\hline & Mentha arvensis L. & Eurasia, California & 9 & & 13 \\
\hline & Mentha longifolia (L.) Huds. & Eurasia, Africa & & 4 & \\
\hline & Mentha pulegium L. & $\begin{array}{l}\text { Eurasia, Africa introduced } \\
\text { from America }\end{array}$ & & 4 & 1 \\
\hline & Mentha spicata L. & $\begin{array}{l}\text { Europe introduced from } \\
\text { California }\end{array}$ & & 4,8 & 10 \\
\hline & Mentha suaveolens Ehrh. & Mediterranean region & & $3,4,10$ & \\
\hline & Mentha $\times$ gentilis $\mathrm{L}$. & Europe & & 8 & \\
\hline & $\begin{array}{l}\text { Mentha } \times \text { piperita L. (M. } \\
\text { aquatica L. X M. spicata L.) }\end{array}$ & $\begin{array}{l}\text { Europe introduced from } \\
\text { California }\end{array}$ & & 4,8 & 10 \\
\hline & Monardella villosa Benth. & California & $1,4,10$ & & \\
\hline & Ocimum basilicum L. & Africa & & 8 & \\
\hline & Origanum majorana L. & Europe & & 6,7 & \\
\hline & Origanum vulgare L. ssp. vulgare & Europe & & $4,8,11$ & \\
\hline & Phlomis lychnitis L. & Europe & & 4 & \\
\hline & Rosmarinus officinalis L. & Europe & & $\begin{array}{l}1,3,4,5,6,7,8 \\
11,14\end{array}$ & $\begin{array}{l}3,4,9,10, \\
11\end{array}$ \\
\hline & Salvia sp. & California, Mexico & 8,11 & 3,5 & 3,8 \\
\hline & Salvia aethiopis L. & Europe & & & 3,8 \\
\hline & $\begin{array}{l}\text { Salvia apiana Jepson [Ramona } \\
\text { polystachya (Benth.) Greene] }\end{array}$ & California & $4,8,9,10$ & & \\
\hline & Salvia carduaceae Benth. & California & 5 & & \\
\hline & Salvia columbariae Benth. & California & $1,3,4,6,9,11$ & & \\
\hline & Salvia lavandulifolia Vahl & Europe & & 8 & \\
\hline & Salvia lavanduloides Kunth & Mexico & & 10 & \\
\hline & Salvia leucantha Cav. & Mexico & & 7,12 & \\
\hline & $\begin{array}{l}\text { Salvia mellifera E. Greene } \\
\text { [Ramona stachyoides (Benth.) Briq.] }\end{array}$ & California & $1,4,8,9,10$ & & 3 \\
\hline & Salvia officinalis L. & Europe & & $1,3,8,10,14$ & \\
\hline & Salvia spathacea E. Greene & California & $1,7,11$ & & \\
\hline & Salvia verbenaca $\mathrm{L}$. & Eurasia, Africa & & $2,4,5,11$ & \\
\hline & $\begin{array}{l}\text { Satureja douglasii (Benth.) Briq. } \\
\text { [Clinopodium douglasii (Benth.) } \\
\text { Kuntze] }\end{array}$ & California & $\begin{array}{l}1,3,4,5,7,8 \\
9,10,11,13\end{array}$ & & $3,4,7,8$ \\
\hline & Satureja hortensis L. & Eurasia & & 3,10 & \\
\hline & $\begin{array}{l}\text { Satureja macrostema (Moc. \& } \\
\text { Sesse ex Benth.) Briq. }\end{array}$ & Mexico & & 4,6 & \\
\hline & Stachys albens A. Gray & California & $3,4,10$ & & \\
\hline & Stachys bullata Benth & California & $3,9,10$ & & \\
\hline & Teucrium chamaedrys L. & Mediterranean region & & 9 & \\
\hline & Teucrium scorodonia L. & Europe, Africa & & 3 & \\
\hline & Thymus sp. & Eurasia, Africa & & 7,11 & \\
\hline & Thymus mastichina (L.) L. & Spain & & 11 & \\
\hline & Thymus praecox Opiz & Europe & & 8 & \\
\hline & Thymus vulgaris $\mathrm{L}$. & Mediterranean region & & $1,2,3,4,6,7,8$ & \\
\hline
\end{tabular}


Table 3 Medicinal plants used before, during and after the Mission period, and present time at Mission Gardens. The numbers refer to emic and etic illness groupings (see Table 2) (Continued)

\begin{tabular}{|c|c|c|c|c|c|}
\hline Botanical family* & Medicinal plants* & Native & $\begin{array}{l}\text { Pre-Mission } \\
\text { period }\end{array}$ & Mission period & $\begin{array}{l}\text { Post- } \\
\text { Mission } \\
\text { period }\end{array}$ \\
\hline & & & & 11,12 & \\
\hline & Thymus zygis $\mathrm{L}$. & Spain, Africa & & 11 & \\
\hline & Trichostema lanatum Benth. & California & $\begin{array}{l}3,4,5,6,7,8 \\
11\end{array}$ & & \\
\hline & Trichostema lanceolatum Benth. & California & $\begin{array}{l}3,4,6,7,9 \\
10,11,13\end{array}$ & & $\begin{array}{l}3,6,8,9 \\
10\end{array}$ \\
\hline \multirow[t]{2}{*}{ Laminariaceae } & Laminaria sp. & California & 4 & & \\
\hline & Macrocystis sp. & California & 4 & & \\
\hline \multirow[t]{2}{*}{ Lauraceae } & Laurus nobilis L. & Europe & & $3,4,7,11$ & \\
\hline & $\begin{array}{l}\text { Umbellularia californica } \\
\text { (Hook. \& Arn.) Nutt. }\end{array}$ & California & $3,4,7,8,10$ & & 8 \\
\hline \multirow[t]{4}{*}{ Liliaceae } & Lilium candidum L. & Balkan Peninsula, Middle East & & 3 & \\
\hline & $\begin{array}{l}\text { Prosartes parvifolia S. Watson } \\
\text { [Disporum hookeri (Torr.) G. } \\
\text { Nicholson] }\end{array}$ & California & 13 & & \\
\hline & $\begin{array}{l}\text { Hesperolinon californicum } \\
\text { (Benth.) Small }\end{array}$ & California & 11 & & \\
\hline & Linum usitatissimum L. & Asia, Africa & & 11 & \\
\hline \multirow[t]{3}{*}{ Loasaceae } & Mentzelia sp. & California & 11 & & \\
\hline & Mentzelia aspera L. & California and Mexico & & 6 & \\
\hline & Mentzelia hispida Willd. & California & & 4,6 & \\
\hline \multirow[t]{2}{*}{ Lythraceae } & Cuphea aequipetala Cav. & Mexico & & 3,4 & \\
\hline & Heimia salicifolia Link. & Mexico & & 3,12 & \\
\hline Magnoliaceae & Magnolia grandiflora L. & Mexico and South West USA & & 1,8 & \\
\hline \multirow[t]{2}{*}{ Malpighiaceae } & Byrsonima crassifolia (L.) Kunth. & Mexico & & 3,4 & \\
\hline & Galphimia glauca Cav. & Mexico & & 3,7 & \\
\hline \multirow[t]{18}{*}{ Malvaceae } & Abutilon palmeri A. Gray & California & & & $1,3,11$ \\
\hline & Alcea rosea $\mathrm{L}$. & $\begin{array}{l}\text { China introduced from } \\
\text { Europe }\end{array}$ & & 8,11 & \\
\hline & Althaea officinalis $L$. & Eurasia, Africa & & 4,11 & \\
\hline & Ceiba pentandra (L.) Gaertn. & Mexico & & $3,4,8$ & \\
\hline & $\begin{array}{l}\text { Chiranthodendron pentadactylon } \\
\text { Larreategui }\end{array}$ & Mexico & & 1,8 & \\
\hline & $\begin{array}{l}\text { Fremontodendron californicum } \\
\text { (Torrey) Cov. }\end{array}$ & California & 10 & & \\
\hline & $\begin{array}{l}\text { Guazuma tomentosa Kunth } \\
\text { (G. Ulmifolia Lam.) }\end{array}$ & Mexico & & $3,4,6,12$ & \\
\hline & Hibiscus sabdariffa L. & West of Africa & & $4,8,12,13$ & \\
\hline & Hibiscus rosa-sinensis $\mathrm{L}$. & West of Africa & & 9,12 & \\
\hline & Malacothamnus sp. (Malvastrum sp.) & California & $4,5,7$ & & \\
\hline & $\begin{array}{l}\text { Malacothamnus fasciculatus } \\
\text { (Torrey \& A. Gray) E. Greene }\end{array}$ & California & 4 & & \\
\hline & Malva moschata L. & Eurasia & & 11 & \\
\hline & Malva neglecta Wallr. & Eurasia & & $3,4,11,14$ & \\
\hline & Malva parviflora $\mathrm{L}$. & Europe & & 3,4 & \\
\hline & Malva sylvestris $\mathrm{L}$. & Europe & & $\begin{array}{l}1,3,4,6,5,7,8 \\
9,10,11,14\end{array}$ & $1,3,11$ \\
\hline & Malvaviscus arboreus Cav. & Mexico & & 4,6 & \\
\hline & Pavonia schiedeana Steud. & Mexico & & $1,3,4,6$ & \\
\hline & $\begin{array}{l}\text { Pseudobombax ellipticum } \\
\text { (Kunth) Dugard }\end{array}$ & Mexico & & 10 & \\
\hline
\end{tabular}


Table 3 Medicinal plants used before, during and after the Mission period, and present time at Mission Gardens. The numbers refer to emic and etic illness groupings (see Table 2) (Continued)

\begin{tabular}{|c|c|c|c|c|c|}
\hline Botanical family* & Medicinal plants* & Native & $\begin{array}{l}\text { Pre-Mission } \\
\text { period }\end{array}$ & Mission period & $\begin{array}{l}\text { Post- } \\
\text { Mission } \\
\text { period }\end{array}$ \\
\hline & Sida acuta Burm. & Mexico & & $3,4,13$ & \\
\hline & Sida rhombifolia L. & Mexico & & 7,8 & \\
\hline & $\begin{array}{l}\text { Sphaeralcea emonyi Torr. } \\
\text { ex A. Gray }\end{array}$ & California & & & 3,9 \\
\hline & Theobroma cacao L. & Mexico, Amazon basin & & 11 & \\
\hline & Tilia sp. & Eurasia, Mexico & & 8 & \\
\hline & Tilia cordata Mill. & Europe & & 8 & \\
\hline & $\begin{array}{l}\text { Tilia platyphyllos Scop. ssp. } \\
\text { platyphyllos }\end{array}$ & Eurasia & & 4,8 & \\
\hline Martyniaceae & Martynia annua L. & Mexico & & 8 & \\
\hline \multirow[t]{3}{*}{ Melanthiaceae } & $\begin{array}{l}\text { Trillium chloropetalum (Torrey) } \\
\text { Howell }\end{array}$ & California & 1,4 & & \\
\hline & $\begin{array}{l}\text { Zigadenus fremontii (Torr.) S. } \\
\text { Watson [Toxicoscordion } \\
\text { fremontii (Torr.) Rydb.] }\end{array}$ & California & 3 & & \\
\hline & $\begin{array}{l}\text { Zigadenus venenosus (S. Watson) } \\
\text { Rydb. [Toxicoxcordion venenosus } \\
\text { (S. Watson) Rydb.] }\end{array}$ & California & 3 & & \\
\hline Melastomataceae & Miconia albicans (Sw.) DC. & Mexico & & 4,13 & \\
\hline Meliaceae & Cedrela odorata L. & Mexico & & $4,9,12$ & \\
\hline Menispermaceae & Cissampelos pareira $\mathrm{L}$. & Africa & & 1,4 & \\
\hline Montiaceae & Claytonia perfoliata Willd. & California & 4 & & \\
\hline \multirow[t]{4}{*}{ Moraceae } & Brosimum alicastrum SW. & Mexico & & $3,10,13$ & \\
\hline & Dorstenia contrajerva $\mathrm{L}$. & Mexico & & 7,12 & \\
\hline & Ficus carica $\mathrm{L}$. & Middle east, western Asia & & 3,11 & \\
\hline & Ficus petiolaris Kunth & Mexico & & $1,4,7$ & \\
\hline Muntingiaceae & Muntingia calabura $\mathrm{L}$. & Mexico & & $3,6,7$ & \\
\hline \multirow[t]{2}{*}{ Musaceae } & Musa sp. & Asia & & 11 & \\
\hline & $\begin{array}{l}\text { Musa sapientum L. (Musa } \\
\text { x paradisiac L.) }\end{array}$ & $\begin{array}{l}\text { Indonesian, grown in } \\
\text { countries with tropical } \\
\text { climate }\end{array}$ & & 4 & 3,11 \\
\hline \multirow[t]{5}{*}{ Myrtaceae } & Eucalyptus sp. & Australia & & & 8 \\
\hline & Eucalyptus globulus Labill. & Australia & & 3,11 & \\
\hline & Eugenia acapulcensis Steud. & Central America & & $4,6,10$ & \\
\hline & Myrtus communis L. & Europe & & & \\
\hline & Psidium guava $\mathrm{L}$. & Central America and Mexico & & 4 & \\
\hline Nyctaginaceae & Abronia sp. & California & 13 & & \\
\hline \multirow[t]{10}{*}{ Oleaceae } & $\begin{array}{l}\text { Forestierra pubescence Nutt. } \\
\text { (F. neomexicana A. Gray) }\end{array}$ & California & & & 8 \\
\hline & $\begin{array}{l}\text { Fraxinus angustifolia Vahl } \\
\text { ssp. angustifolia }\end{array}$ & Europe & & 2 & \\
\hline & Fraxinus dipetala Hook. \& Arn. & California & 3,11 & & \\
\hline & Fraxinus excelsior $\mathrm{L}$. & & & 1,3 & \\
\hline & Fraxinus latifolia Benth. & California & 11 & & 11 \\
\hline & Fraxinus uhdei (Wenz.) Lingel. & Mexico & & 12 & \\
\hline & Jasminum officinale L. & Middle East, India, China & & & $4,6,8$ \\
\hline & Ligustrum parteri Coult. \& Rose & Europe & & 1,3 & \\
\hline & Olea europaea L. var. europaea & Mediterranea region & & $1,3,4,13$ & \\
\hline & Olea europaea L. var. sylvestris (Mill.) Brot. & Mediterranea region & & 1 & \\
\hline \multirow[t]{2}{*}{ Onagraceae } & Ludwigia octovalvis (Jacq.) P. H. Ravens & Central America & & 3 & \\
\hline & Epilobium canum (E. Greene) Raven & California & $3,7,11,13$ & & \\
\hline
\end{tabular}


Table 3 Medicinal plants used before, during and after the Mission period, and present time at Mission Gardens. The numbers refer to emic and etic illness groupings (see Table 2) (Continued)

\begin{tabular}{|c|c|c|c|c|c|}
\hline Botanical family* & Medicinal plants* & Native & $\begin{array}{l}\text { Pre-Mission } \\
\text { period }\end{array}$ & Mission period & $\begin{array}{l}\text { Post- } \\
\text { Mission } \\
\text { period }\end{array}$ \\
\hline & (Zauschneria californica C. Presl) & & & & \\
\hline & $\begin{array}{l}\text { Gaura coccinea Nutt. Ex Pursh } \\
\text { [Oenothera suffrutescens (Ser.) } \\
\text { W. L. Wagner \& Hoch] }\end{array}$ & California & & & 1,4 \\
\hline & Oenothera albicaulis Pursh & Mexico, West USA & & & 11 \\
\hline & Oenothera elata Kuth & California & & & \\
\hline & Oenothera hookeri Torrey \& A. Gray & California & & & \\
\hline & Oenothera rosea L'Her. Ex Aiton & Mexico and Texas & & 3,4 & \\
\hline \multirow[t]{6}{*}{ Orobanchaceae } & Castilleja sp. & California & 3 & & \\
\hline & Castilleja affinis Hook. \& Arn. & California & 3 & & \\
\hline & $\begin{array}{l}\text { Castilleja attenuata (A. Gray) } \\
\text { Chuang \& Heckard }\end{array}$ & California & 10 & & \\
\hline & Castilleja elastica Sesse ex Cerv. & Mexico & & 7,12 & \\
\hline & Castilleja tenuiflora Benth. & Mexico and South West USA & & $1,3,7,12$ & \\
\hline & Orthocarpus sp. & California & 10 & & \\
\hline \multirow[t]{2}{*}{ Paeoniaceae } & Paeonia brownii Hook. & California & $1,4,10$ & & \\
\hline & Paeonia californica Torrey \& A. Gray & California & $\begin{array}{l}1,3,4,5,7,8 \\
10,11,13\end{array}$ & & $3,4,8,10$ \\
\hline \multirow[t]{8}{*}{ Papaveraceae } & $\begin{array}{l}\text { Argemone mexicana } \mathrm{L} \text {. } \\
\text { (A. sanguinea Greene) }\end{array}$ & Mexico & & $3,7,9,12,13$ & \\
\hline & Chelidonium majus L. & Eurasia & & $1,3,8,11$ & \\
\hline & Eschscholzia sp. & California & 8 & & \\
\hline & Eschscholzia californica Cham. & California & $3,4,8,9$ & & \\
\hline & Fumaria officinalis L. ssp. officinalis & Eurasia, Africa & & 3 & \\
\hline & Papaver rhoeas L. & Eurasia, Africa & & $4,8,11$ & \\
\hline & Papaver somniferum $\mathrm{L}$. & $\begin{array}{l}\text { Eastern Mediterranean, } \\
\text { introduced from Eurasia }\end{array}$ & & 8 & \\
\hline & Romneya coulteri Harv. & California & $3,4,9$ & & \\
\hline Pelliaceae & Pellia californica Cham. & California & 11 & & \\
\hline \multirow[t]{2}{*}{ Petiveraceae } & Petiveria alliacea $\mathrm{L}$. & Mexico & & $3,8,12$ & \\
\hline & Rivina humilis $\mathrm{L}$. & Mexico & & $3,4,12$ & \\
\hline \multirow[t]{3}{*}{ Phrymaceae } & $\begin{array}{l}\text { Mimulus aurantiacus Curtis } \\
\text { (M. puniceus Nutt.) }\end{array}$ & California & $3,5,13$ & & \\
\hline & $\begin{array}{l}\text { Mimulus glutinosus J. C. Wendl. } \\
\text { (M. aurantiacus Torr.) }\end{array}$ & California & & & \\
\hline & Mimulus guttatus DC & California & 4 & & \\
\hline Picrodendraceae & Petalostigma pubescens Domin & Australia, New Guinea & & & 10,11 \\
\hline \multirow[t]{9}{*}{ Pinaceae } & $\begin{array}{l}\text { Abies concolor (Gordon \& } \\
\text { Glend.) Lindley }\end{array}$ & California & & & 4,6 \\
\hline & Pinus sp. & California & $\begin{array}{l}1,3,7,8,9 \\
10,11\end{array}$ & $1,3,4,11$ & 7,8 \\
\hline & Pinus halepensis Mill. & Mediterranean region & & $3,11,12$ & \\
\hline & $\begin{array}{l}\text { Pinus monophylla Torrey \& } \\
\text { Fremont }\end{array}$ & California & 10,11 & & \\
\hline & Pinus patula Schiede ex Schltdl. \& Cham. & Mexico & & 10 & \\
\hline & Pinus pinaster Aiton. & Europe & & 3 & \\
\hline & Pinus sabiniana Douglas & California & 3,7 & & \\
\hline & Pinus sylvestris L. & Eurasia & & 11 & \\
\hline & Pseudotsuga menziesii (Mirb.) Franco & California & & & $6,11,13$ \\
\hline \multirow[t]{2}{*}{ Piperaceae } & Peperomia pellucida Kunth & South and Central America & & 3,4 & \\
\hline & Piper sanctum (Miq.) Schltdl. Ex C. DC. & Mexico & & 8 & \\
\hline
\end{tabular}


Table 3 Medicinal plants used before, during and after the Mission period, and present time at Mission Gardens. The numbers refer to emic and etic illness groupings (see Table 2) (Continued)

\begin{tabular}{|c|c|c|c|c|c|}
\hline Botanical family* & Medicinal plants* & Native & $\begin{array}{l}\text { Pre-Mission } \\
\text { period }\end{array}$ & Mission period & $\begin{array}{l}\text { Post- } \\
\text { Mission } \\
\text { period }\end{array}$ \\
\hline \multirow[t]{12}{*}{ Plantaginaceae } & Antirrhinum nuttallianum Benth. & California & 10 & & \\
\hline & Digitalis minor $\mathrm{L}$. & Spain's Balearic Islands & & 1 & \\
\hline & Digitalis purpurea $\mathrm{L}$. & Europe & & & \\
\hline & Globularia alypum L. & Mediterranean region & & 1 & \\
\hline & Keckiella antirrhinoides (Benth.) Straw & California & & & \\
\hline & Keckiella breviflora (Lindley) Straw & California & 3,10 & & \\
\hline & $\begin{array}{l}\text { Keckiella cordifolia (Benth.) Straw } \\
\text { (Penstemon cordifolius Benth.) }\end{array}$ & California & 3,10 & & \\
\hline & Penstemon centranthifolius Benth. & California & 3 & & \\
\hline & Plantago sp. & California & $1,3,9,10,11$ & & \\
\hline & Plantago lagopus L. & Eurasia, Africa & & $5,11,12$ & \\
\hline & Plantago lanceolata $\mathrm{L}$. & Eurasia & & $\begin{array}{l}3,4,5,7,11,12 \\
14\end{array}$ & \\
\hline & Plantago major $\mathrm{L}$. & Eurasia & & $\begin{array}{l}1,3,7,11,12 \\
14\end{array}$ & $4,9,11$ \\
\hline \multirow[t]{3}{*}{ Platanaceae } & Platanus lindeliana Mart. \& Gal. & Mexico & & $7,8,10$ & \\
\hline & Platanus racemosa Nutt. & California & 10,11 & & \\
\hline & Platanus x hispanica Mill. ex Münch. & Europe & & 1 & \\
\hline \multirow[t]{2}{*}{ Plumbaginaceae } & Limonium californicum (Boiss.) A. A. Heller & California & $1,6,10,11$ & & \\
\hline & Plumbago pulchella Boiss. & Mexico & & $3,6,12$ & \\
\hline \multirow[t]{12}{*}{ Poaceae } & Arundo donax L. & Mediterranean region, Asia & & 2,10 & \\
\hline & Avena sativa $\mathrm{L}$. & $\begin{array}{l}\text { Europe and naturalized in } \\
\text { California }\end{array}$ & & 4 & \\
\hline & Bouteloua eriopoda (Torrey) Torrey & California & & & \\
\hline & Coix lachrymal-jobi L. & Asia & & 1 & \\
\hline & Cynodon dactylon (L.) Pers. & Africa & & 4 & \\
\hline & Distichlis spicata (L.) E. Greene & California & $1,3,4,6,8,10$ & & \\
\hline & $\begin{array}{l}\text { Elymus condensatus (J. Presl) A. } \\
\text { Love (Leymus condensatus J. Presl) }\end{array}$ & California & $4,6,11$ & & \\
\hline & Elymus repens (L.) Gould & Europe & & & 6,13 \\
\hline & Oryza sp. & Asia and Europe & & & 3,4 \\
\hline & Oryza sativa L. & $\begin{array}{l}\text { Africa, Asia, introduced from } \\
\text { all continents }\end{array}$ & & 4 & 3,4 \\
\hline & Triticum aestivum $\mathrm{L}$. & Europe & & $\begin{array}{l}3,4,5,7,9,11 \\
14\end{array}$ & \\
\hline & Zea mays L. & Mexico & & $1,2,3,4,11,12$ & 3,11 \\
\hline \multirow[t]{2}{*}{ Polemoniaceae } & Loeselia mexicana (Lam.) Brand & Mexico & & 3 & 1,4 \\
\hline & $\begin{array}{l}\text { Navarretia atractyloides (Benth.) } \\
\text { Hook. \& Arn. }\end{array}$ & California & 3 & & \\
\hline \multirow[t]{9}{*}{ Polygonaceae } & Chorizanthe sp. & California & 3,11 & & \\
\hline & Eriogonum sp & California & $4,5,8,9$ & & \\
\hline & Eriogonum elongatum Benth. & California & $1,10,11$ & & \\
\hline & Eriogonum fasciculatum Benth. & California & $4,5,7,8,9$ & & $3,6,8,10$ \\
\hline & $\begin{array}{l}\text { Eriogonum nudum Benth. [E. } \\
\text { latifolium Smith ssp. nudum } \\
\text { (Douglas ex Bentham) S. Stokes] }\end{array}$ & California & $1,3,10,11$ & & 10 \\
\hline & Rheum rhabarbarum L. & $\begin{array}{l}\text { Asia, introduced to California } \\
\text { from Europe }\end{array}$ & & & 7 \\
\hline & Rumex sp. & California & & & \\
\hline & Rumex crispus L. & Eurasia & & & $4,6,11$ \\
\hline & Rumex hymenosepalus Torrey & California & $3,4,7,8,10$ & & $4,8,10$ \\
\hline
\end{tabular}


Table 3 Medicinal plants used before, during and after the Mission period, and present time at Mission Gardens. The numbers refer to emic and etic illness groupings (see Table 2) (Continued)

\begin{tabular}{|c|c|c|c|c|c|}
\hline Botanical family* & Medicinal plants* & Native & $\begin{array}{l}\text { Pre-Mission } \\
\text { period }\end{array}$ & Mission period & $\begin{array}{l}\text { Post- } \\
\text { Mission } \\
\text { period }\end{array}$ \\
\hline & & & 11 & & \\
\hline & Rumex obtusifolius L. & Europe & & $1,3,4$ & \\
\hline \multirow[t]{2}{*}{ Polypodiaceae } & Phlebodium aureum (L.) J. Sm. & South and Central America & & 4,13 & \\
\hline & Polypodium californicum Kaulf. & California & $1,3,7,11$ & & \\
\hline Portulacaceae & Portulaca oleracea $\mathrm{L}$. & $\begin{array}{l}\text { Eurasia, introduced to } \\
\text { Mexico }\end{array}$ & & 1 & \\
\hline \multirow[t]{4}{*}{ Primulaceae } & $\begin{array}{l}\text { Anagallis arvensis L. [Lysimachia } \\
\text { arvensis (L.) U. Manns \& Anderb.] }\end{array}$ & Europe & 3,6 & $1,3,6,11$ & \\
\hline & $\begin{array}{l}\text { Anagallis foemina Mill. (Lysimachia } \\
\text { foemina Mill.) }\end{array}$ & Europe & & 6 & \\
\hline & Primula elatior L. ssp. elatior & Europe & & 7 & \\
\hline & Primula veris $\mathrm{L}$. & Eurasia & & 3 & \\
\hline \multirow[t]{7}{*}{ Pteridaceae } & $\begin{array}{l}\text { Adiantum aleuticum (Rupr.) C.A. } \\
\text { Paris (A. pedatum L.) }\end{array}$ & California, Europe, Mexico & $1,4,7$ & & \\
\hline & Adiantum capillus-veneris $\mathrm{L}$. & California & & $\begin{array}{l}3,4,5,10,11 \\
13\end{array}$ & $1,3,4,7$ \\
\hline & Adiantum jordanii Mueller & California & $1,4,5,7,8,11$ & & 1,4 \\
\hline & Pellaea andromedifolia (Kaulf.) Fee & California & $1,4,5,7$ & & \\
\hline & Pellaea atropurpurea (L.) Link & Mexico & & & $3,11,13$ \\
\hline & $\begin{array}{l}\text { Pellaea mucronata (D. Eaton) D. } \\
\text { Eaton (P. ornithopus Hook.) }\end{array}$ & California & $1,3,4,11$ & & \\
\hline & $\begin{array}{l}\text { Pentagramma triangularis (Kaulf.) } \\
\text { G. Yatskievych, Windhan \& } \\
\text { Wollenweber }\end{array}$ & California & 3,7 & & \\
\hline \multirow[t]{10}{*}{ Ranunculaceae } & Actaea rubra (Aiton) Willd. & California & & & 11 \\
\hline & Aquilegia sp. & California & & & 4 \\
\hline & Aquilegia truncate Fisch. ex DC. & California & & & \\
\hline & Clematis lasiantha Nutt. & California & 3,6 & & \\
\hline & Clematis ligusticifolia Nutt. & California & $1,3,6,10$ & & $1,3,10,11$ \\
\hline & Clematis pauciflora Nutt. & California & $3,10,11,13$ & & \\
\hline & Clematis virginiana L. & Eastern U.S.A. & & & \\
\hline & $\begin{array}{l}\text { Helleborus viridis L. ssp. occidentalis } \\
\text { (Reut.) Schiffn. }\end{array}$ & Europe & & 4 & \\
\hline & Ranunculus sp. & California & 3 & & \\
\hline & Ranunculus ficaria L. & Eurasia & & 3 & \\
\hline Resedaceae & Reseda alba $\mathrm{L}$. & Eurasia, Africa & & 4 & \\
\hline \multirow[t]{11}{*}{ Rhamnaceae } & Ceanothus sp. & California & 3 & & \\
\hline & Ceanothus arboreus Greene & California & & & \\
\hline & Ceanothus leucodermis Greene & California & & & 7 \\
\hline & Ceanothus thyrsiflorus Eschsch. & California & & & \\
\hline & Ceanothus verrucosus Nutt. & California & & & \\
\hline & $\begin{array}{l}\text { Frangula californica (Eschsch.) A. } \\
\text { Gray ssp. occidentalis (Rhamnus } \\
\text { californica Eschsch.) }\end{array}$ & California & $1,3,4,6,7,11$ & & \\
\hline & $\begin{array}{l}\text { Frangula purshiana (DC.) Cooper } \\
\text { (Rhamnus purshiana DC.) }\end{array}$ & California & 4 & & \\
\hline & Gouania polygama (Jacq.) Urb. & Mexico & & $6,8,10$ & \\
\hline & $\begin{array}{l}\text { Karwinskia humboldtiana (Schult.) } \\
\text { Zucc. (Rhamnus humboldtiana Schult.) }\end{array}$ & Mexico and Texas & & 8 & \\
\hline & Rhamnus alaternus $\mathrm{L}$. & Mediterranean region & & 1,11 & \\
\hline & $\begin{array}{l}\text { Rhamnus californica Eschsch. } \\
\text { [Frangula californica (Eschsch.) A. Gray] }\end{array}$ & California & $3,4,7$ & & 4 \\
\hline
\end{tabular}


Table 3 Medicinal plants used before, during and after the Mission period, and present time at Mission Gardens. The numbers refer to emic and etic illness groupings (see Table 2) (Continued)

\begin{tabular}{|c|c|c|c|c|c|}
\hline Botanical family* & Medicinal plants* & Native & $\begin{array}{l}\text { Pre-Mission } \\
\text { period }\end{array}$ & Mission period & $\begin{array}{l}\text { Post- } \\
\text { Mission } \\
\text { period }\end{array}$ \\
\hline & Rhamnus crocea Nutt. & California & & & \\
\hline & Rhamnus ilicifolia Kellogg & California & 6,10 & & \\
\hline Rhizophoraceae & Rhizophora mangle L. & Mexico & & $1,6,13$ & \\
\hline Rhodomelaceae & Alsidium helminthochorton (Schw.) Kütz. & Not documented & & 4 & \\
\hline \multirow[t]{33}{*}{ Rosaceae } & Adenostoma sp. & California & $3,4,7,8$ & & \\
\hline & Adenostoma fasciculatum Hook. \& Arn. & California & $3,5,7,11$ & & \\
\hline & Adenostoma sparsifolium Torr. & California & $\begin{array}{l}1,3,4,6,8,9 \\
10,11\end{array}$ & & \\
\hline & Agrimonia eupatoria L. ssp. euptoria & Europe & & 4,11 & \\
\hline & Chamaebatia foliolosa Benth. & California & & & 3,4 \\
\hline & Cercocarpus betuloides Torrey \& A. Gray & California & 4,10 & & \\
\hline & Crataegus monogyna Jacq. & Eurasia, Africa & & $1,3,4,8,11,14$ & \\
\hline & Cydonia oblonga Mill. & $\begin{array}{l}\text { Asia introduced to California } \\
\text { from Europe }\end{array}$ & & $4,8,9$ & \\
\hline & Eriobotrya japonica (Thunb.) Lindl. & Asia & & 4 & \\
\hline & $\begin{array}{l}\text { Heteromeles arbutifolia (Lindley) } \\
\text { Roemer (Photinia arbutifolia Lindl.) }\end{array}$ & California & 3,5 & & \\
\hline & Horkelia cuneata Lindl. & California & $\begin{array}{l}1,4,6,8,10 \\
11\end{array}$ & & \\
\hline & Malus domestica (Borkh.) Borkh. & $\begin{array}{l}\text { Asia introduced from } \\
\text { Europe, North America }\end{array}$ & & $1,3,4,8,11$ & \\
\hline & Malus sylvestris Mill. & Europe & & 4 & \\
\hline & $\begin{array}{l}\text { Potentilla glandulosa Lindl. } \\
\text { [Drymocallis glandulosa (Lindl.) Rydb.] }\end{array}$ & California & $\begin{array}{l}1,4,6,8,10 \\
11\end{array}$ & & \\
\hline & Potentilla reptans $\mathrm{L}$. & Eurasia, Africa & & $1,3,13$ & \\
\hline & Prunus avium (L.) L. & Eurasia & & $2,4,12$ & \\
\hline & Prunus cerasus L. & Eurasia & & 4,10 & \\
\hline & Prunus domestica $\mathrm{L}$. & Asia & & 4 & \\
\hline & Prunus dulcis (Mill.) D.A. Webb & Asia & & 11 & \\
\hline & Prunus emarginata (Hook.) Walp. & California & $3,7,11$ & & \\
\hline & $\begin{array}{l}\text { Prunus ilicifolia (Nutt. Ex Hook. \& Arn.) } \\
\text { Walp. [Cerasus ilicifolia Nutt. Ex } \\
\text { Hook \& Arn.] }\end{array}$ & California & 4,10 & & \\
\hline & Prunus integrifolia (C. Presl) Walp. & South America & & & \\
\hline & Prunus serotina Ehrh. & Mexico, South West USA & & 10 & \\
\hline & Prunus spinosa $\mathrm{L}$. & Eurasia & & $1,3,4,10,11$ & \\
\hline & $\begin{array}{l}\text { Prunus virginiana } \mathrm{L} \text {. var. demissa } \\
\text { (Nutt.) Torrey [Cerasus virginana } \\
\text { (L.) Michx.] }\end{array}$ & California & 4,10 & & \\
\hline & Rosa sp. & Eurasia & & 9 & \\
\hline & Rosa agrestis Savi & Europe & & 4,14 & \\
\hline & Rosa californica Cham. \& Schldl. & California & $\begin{array}{l}3,4,7,9,10 \\
11,13\end{array}$ & & 9 \\
\hline & Rosa canina L. & Eurasia, Africa & & 4,11 & \\
\hline & Rosa gallica $\mathrm{L}$. & Eurasia & & & 4,9 \\
\hline & Rubus ulmifolius Schott & $\begin{array}{l}\text { Europe, Africa, introduced } \\
\text { from California }\end{array}$ & & $1,3,4,5,7,11$ & \\
\hline & $\begin{array}{l}\text { Rubus ursinus Cham. \& Schldl } \\
\text { (R. vitifolius Cham. \& Schldl.) }\end{array}$ & California & $3,4,5,6$ & & 4 \\
\hline & Sorbus domestica $\mathrm{L}$. & Eurasia, Africa & & 4 & \\
\hline Rubiaceae & Cinchona officinalis $\mathrm{L}$. & South America & & & 10 \\
\hline
\end{tabular}


Table 3 Medicinal plants used before, during and after the Mission period, and present time at Mission Gardens. The numbers refer to emic and etic illness groupings (see Table 2) (Continued)

\begin{tabular}{|c|c|c|c|c|c|}
\hline Botanical family* & Medicinal plants* & Native & $\begin{array}{l}\text { Pre-Mission } \\
\text { period }\end{array}$ & Mission period & $\begin{array}{l}\text { Post- } \\
\text { Mission } \\
\text { period }\end{array}$ \\
\hline & Coffea arabica L. & Africa & & $4,7,8,11$ & \\
\hline & Galium angustifolium Nutt. & California & 4 & & \\
\hline & Galium triflorum Michaux & California & $3,4,7$ & & \\
\hline & Hamelia patens Jacq. & Mexico & & 3,12 & \\
\hline \multirow{8}{*}{ Rutaceae } & Amyris madrensis S. Watson & Mexico & & 3 & \\
\hline & Amyris texana (Buckley) P. Wilson & Mexico & & 3,7 & \\
\hline & Casimiroa edulis La Llave \& Lex. & Mexico & & 1,8 & \\
\hline & Citrus sp. & $\begin{array}{l}\text { Australia, introduced from } \\
\text { Europe }\end{array}$ & & & \\
\hline & Citrus limon (L.) Burm fil. (pro. sp.) & $\begin{array}{l}\text { Asia, introduced from } \\
\text { Europe }\end{array}$ & & $\begin{array}{l}1,3,4,5,6,8 \\
11\end{array}$ & 7,11 \\
\hline & Citrus sinensis L. Osbeck & $\begin{array}{l}\text { Asia, introduced from } \\
\text { Europe }\end{array}$ & & $4,8,11,14$ & $7,8,10$ \\
\hline & Ruta chalepensis L. & Eurasia, Africa & & $1,4,10$ & \\
\hline & Ruta graveolens L. & Europe & & & 8,9 \\
\hline \multirow[t]{7}{*}{ Salicaceae } & $\begin{array}{l}\text { Populus balsamifera L. ssp. } \\
\text { trichocarpa (Torrey \& A. Gray) } \\
\text { Brayshaw (P. trichocarpa Hook.) }\end{array}$ & California & 3,7 & & 3 \\
\hline & Populus fremontii S. Watson & California & $3,7,8$ & & 3,11 \\
\hline & Populus tremuloides Michaux & California & & & 3 \\
\hline & Salix sp. & California & 3,10 & & \\
\hline & Salix exigua Nutt. & California & 3,8 & & \\
\hline & Salix laevigata Bebb & California & 4,11 & & \\
\hline & Salix lasiolepis Benth & California & $1,6,8,10,11$ & & \\
\hline Salviniaceae & Salvinia minima Baker & Mexico & & & 4 \\
\hline \multirow[t]{9}{*}{ Santalaceae } & Arceuthobium sp. & California & & & 3 \\
\hline & Phoradendron californicum Nutt. & California & & $3,4,6$ & \\
\hline & $\begin{array}{l}\text { Phoradendron juniperinum } \\
\text { Engelm. Ex A. Gray }\end{array}$ & California & 3,9 & & \\
\hline & $\begin{array}{l}\text { Phoradendron macrophyllum } \\
\text { (Engelm.) Cockerell }\end{array}$ & California & $5,7,9$ & & \\
\hline & $\begin{array}{l}\text { Phoradendron serotinum (Raf.) } \\
\text { M. C. Johnst. spp. macrophyllum } \\
\text { (Engelm.) Kuijt }\end{array}$ & California & 5,7 & & \\
\hline & $\begin{array}{l}\text { Phoradendron serotinum (Raf.) M. C. } \\
\text { Johnst. ssp. tomentosum (DC.) Kuijt } \\
\text { [P. leucarpum (Raf.) Reveal \& M. C. } \\
\text { Johnst. ssp. tomentosum (DC.) J. R. Abbott \& R. L. Thomps.; P. coloradensa Raf.] }\end{array}$ & California & $3,7,11$ & & \\
\hline & Phoradendron villosum Nutt. & California & $3,5,7,9$ & & \\
\hline & Phoradendron villosum Nutt. [P. flavescens (Pursh.) Nutt.] & California & $5,7,9$ & & \\
\hline & Viscum album L. ssp. album & Eurasia & & 1,10 & \\
\hline \multirow[t]{3}{*}{ Sapindaceae } & Aesculus californica (Spach) Nutt. & California & $1,3,8,9$ & & 1 \\
\hline & Aesculus hippocastanum L. & Europe & & $1,7,13$ & \\
\hline & Dodonaea viscosa Jacq. & Mexico & & $3,4,6,7,12$ & \\
\hline \multirow[t]{2}{*}{ Sapotaceae } & Achras zapota L. [Manilkara zapota (L.) P. Royen] & Mexico & & $4,7,8,9,12,13$ & \\
\hline & Manilkara sp. & Mexico & & & 3 \\
\hline Sarraceniaceae & Darlingtonia californica Torr. & California & & & \\
\hline Saururaceae & Anemopsis californica (Nutt.) Hook. \& Arn. & California, Mexico & $\begin{array}{l}1,2,3,6,7,8 \\
10,11,13\end{array}$ & $3,5,7,11$ & $1,3,7,10$ \\
\hline \multirow[t]{2}{*}{ Scrophulariaceae } & Buddleja americana L. & Mexico & & 13 & \\
\hline & Capraria biflora L. & Mexico & & 10 & \\
\hline
\end{tabular}


Table 3 Medicinal plants used before, during and after the Mission period, and present time at Mission Gardens. The numbers refer to emic and etic illness groupings (see Table 2) (Continued)

\begin{tabular}{|c|c|c|c|c|c|}
\hline Botanical family* & Medicinal plants* & Native & $\begin{array}{l}\text { Pre-Mission } \\
\text { period }\end{array}$ & Mission period & $\begin{array}{l}\text { Post- } \\
\text { Mission } \\
\text { period }\end{array}$ \\
\hline & Russelia sarmentosa Jaca. & Europe & & $3,4,8$ & \\
\hline & Scrophularia alpestris Gay ex Benth. & Europe & & 3 & \\
\hline & Scrophularia balbisii Hornem. ssp. balbisii & Eurasia, North America & & 3,7 & \\
\hline & Scrophularia californica Cham. \& Schldl. & California & $3,6,9,11$ & & \\
\hline & Verbascum sinuatum $\mathrm{L}$. & Eurasia, Africa & & $1,3,11,13$ & \\
\hline & Verbascum thapsus L. & Europe & & 9,11 & 4 \\
\hline Selaginellaceae & Selaginella lepidophylla (Hook. \& Grev.) Spring & Mexico & & 12,13 & \\
\hline \multirow[t]{2}{*}{ Simaroubaceae } & Castela texana (Torr. \& A. Gray) Rose & Mexico and Texas & & 4 & \\
\hline & Castela tortuosa Liebm. & Mexico & & 6 & \\
\hline Simmondsiaceae & Simmondsia chinensia (Link) C.K.Schneid. & California, Mexico & & & $3,7,11$ \\
\hline \multirow[t]{2}{*}{ Smilacaceae } & Smilax ornata Lem. & Mexico & & & 1,10 \\
\hline & Smilax lanceolata $\mathrm{L}$. & Southeastern USA & & $1,3,4,7,10,12$ & \\
\hline \multirow[t]{20}{*}{ Solanaceae } & Atropa belladonna $\mathrm{L}$. & $\begin{array}{l}\text { Europe, naturalized in } \\
\text { California }\end{array}$ & & & \\
\hline & Capsium annuum L. & Mexico & & & \\
\hline & Datura innoxia Mill. & California & & & 10 \\
\hline & Datura stramonium L. & $\begin{array}{l}\text { Mexico introduced from } \\
\text { Europe }\end{array}$ & & $3,7,10,11,12$ & \\
\hline & Datura wrightii Regel & California & $\begin{array}{l}1,3,4,7,8,9 \\
10,11\end{array}$ & & \\
\hline & Hyoscyamus albus L. & Eurasia & & $4,8,11$ & \\
\hline & Nicotiana sp. & California & $\begin{array}{l}3,4,5,6,7,9 \\
10,11\end{array}$ & & \\
\hline & Nicotiana attenuata Torrey & California & $3,4,6$ & & \\
\hline & Nicotiana clevelandii A. Gray & California & 11 & & \\
\hline & Nicotiana glauca Graham & South America & & $7,8,10,12$ & \\
\hline & Nicotiana quadrivalis Pursh (N. bigelovii Torr.) & California & $\begin{array}{l}3,4,8,9,10 \\
11\end{array}$ & & \\
\hline & Nicotiana pusilla Blanco. (N. rustica L.) & Mexico & & $7,8,10,12$ & \\
\hline & Nicotiana tabacum L. & Mexico & & & $4,8,10$ \\
\hline & Solanum sp. & California & & & 3 \\
\hline & Solanum carolinense L. & USA & & & \\
\hline & Solanum douglasii Dunal & California & $3,9,11$ & & \\
\hline & Solanum lycopersicum L. & Central and South America & & 3 & \\
\hline & Solanum melongena $\mathrm{L}$. & Asia & & 4 & \\
\hline & Solanum nigrum L. & California & $3,6,9$ & & \\
\hline & Solanum tuberosum L. & South America & & 3,7 & 3 \\
\hline Sterculiaceae & Waltheria americana $L$. & Mexico & & $3,10,12$ & \\
\hline Tropaeolaceae & Tropaeolum majus L. & California & & & 11 \\
\hline Turneraceae & Turnera diffusa Willd. ex Schult. & $\begin{array}{l}\text { Southern Texas, Mexico, } \\
\text { South America, Caribbean }\end{array}$ & & $7,8,10,11$ & 7,10 \\
\hline Typhaceae & Typha latifolia L. & California & 1 & & \\
\hline \multirow[t]{6}{*}{ Urticaceae } & Cecropia obtusifolia Bertol. & Mexico & & 4,13 & \\
\hline & Parietaria judaica L. & Eurasia, Africa & & $1,3,4,12$ & \\
\hline & Urtica sp. & California & $1,3,5,7,8$ & & \\
\hline & Urtica dioica $\mathrm{L}$. & Eurasia, Africa & & $\begin{array}{l}1,2,3,4,5,6,7 \\
8,11,14\end{array}$ & \\
\hline & Urtica dioica L. ssp. holosericea (Nutt.) Thorne & California & $7,8,10,11$ & & 4,6 \\
\hline & Urtica urens L. & Eurasia & & $1,3,7$ & \\
\hline
\end{tabular}


Table 3 Medicinal plants used before, during and after the Mission period, and present time at Mission Gardens. The numbers refer to emic and etic illness groupings (see Table 2) (Continued)

\begin{tabular}{|c|c|c|c|c|c|}
\hline Botanical family* & Medicinal plants* & Native & $\begin{array}{l}\text { Pre-Mission } \\
\text { period }\end{array}$ & Mission period & $\begin{array}{l}\text { Post- } \\
\text { Mission } \\
\text { period }\end{array}$ \\
\hline Ustilaginaceae & Ustilago maydis (Persoon) Roussel & Mexico & & 7 & \\
\hline \multirow[t]{5}{*}{ Verbenaceae } & Aloysia citrodora Palau & $\begin{array}{l}\text { South America introduced } \\
\text { from Europe }\end{array}$ & & 4,8 & 1,10 \\
\hline & Aloysia triphylla (L'Her.) Britton & $\begin{array}{l}\text { South America introduced } \\
\text { from Europe }\end{array}$ & & 4,8 & \\
\hline & $\begin{array}{l}\text { Verbena bipinnatifida Nutt. [Glandularia } \\
\text { bipinnatifida (Nutt.) Nutt.] }\end{array}$ & Mexico & & & 10 \\
\hline & Verbena lasiostachys Link var. lasiostachys & California & $3,4,6,11$ & & \\
\hline & Verbena officinalis $\mathrm{L}$. & Europe & & $\begin{array}{l}1,3,4,6,7,11 \\
14\end{array}$ & \\
\hline \multirow[t]{2}{*}{ Violaceae } & Viola sp. & California & 3 & & 6 \\
\hline & Viola riviniana Rchb. & Europe & & 1 & \\
\hline \multirow[t]{2}{*}{ Vitaceae } & Vitis sp. & California & 1 & & \\
\hline & Vitis vinifera $\mathrm{L}$. & Europe & & $1,3,4,7,10,11$ & 7,10 \\
\hline Zosteraceae & Phyllospadix torreyi S. Wats. & California & 11 & & \\
\hline \multirow[t]{4}{*}{ Zygophyllaceae } & Guaiacum officinale L. & Caribbean & & & 3,10 \\
\hline & Guaiacum sanctum L. & Mexico & & $4,6,10$ & \\
\hline & Kallstroemia grandiflora A. Gray & Mexico and South West USA & & $3,7,12$ & \\
\hline & Larrea tridentata (DC.) Cov. (L. californica DC.; L. mexicana Moric) & California, Mexico & $\begin{array}{l}1,3,4,6,7,8, \\
10,11\end{array}$ & $\begin{array}{l}3,4,5,6,7,11 \\
13\end{array}$ & \\
\hline
\end{tabular}

*Botanical family classification and nomenclature for species names were authenticated according to Hickman [45], Stevens [46], and the International Plant Names Index (www.ipni.org)

Rorippa nasturtium-aquaticum (L.) Hayek, Salvia sp. and Sambucus mexicana C. Presl. (Table 3). It is important to point out that these medicinal plants were not necessarily used to treat the same ailments. It is evident that many of the Mission priests and early Spanish explorers were open to the use of Native American medicinal plants and adopted them when medicinal supplies from Spain and Mexico were not available [26, 58-61].

The close reading of diaries, journals, reports, and books indicate there are reasons to believe that sharing of information about medicinal plants did take place at the Missions, but conditions at the Missions and other factors also interfered with the exchange. Table 4 summarizes references that report on the sharing of information. The primary support comes from diaries and reports of priests and others present during the Mission period who observed the use of plants native to California and the introduction of European species period (see Table 4). Direct evidence of the sharing of information comes from reports that neophytes were sent out to collect both food and medicinal plant in times of shortages [64]. A survey conducted in 1812 asked the priests at each Mission to report on the customs and conditions of indigenous people living at or near the Missions [23]. Question no. 15 of the survey asked specifically about the medicinal practices of the people and their use of plants in the treatment of illness. In response to this question, the priest at 13 of the 18 missions reported that the local Native Americans used plants for medicinal purposes. Reports from the other five missions stated that no plants were used by the Native Americans for medicinal purposes. Plant species were identified, ranging in number from one to 14 , at eight of the 13 missions reporting the use of medicinal herbs. A total of 16 different plants were reported from all the California Missions.

Table 5 summarizes references that suggest eight reasons for the impediments to the transfer of information. These are the following:

(1) A significant power imbalance existed between the priests and the Native Americans.

(2) Priests thought the Native Americans were savage heathens or children who knew nothing.

(3) Language barriers to communication.

(4) Reduction in the availability of medicinal herbs due to the elimination of Native American burning and the introduction of Spanish livestock.

(5) Knowledge of medicinal plants was a source of power and income for the Native American shamans who did not want to share it.

(6) Structural organization of the administration of Missions left little time for direct communication between priests and neophytes.

(7) Knowledge of herbal medicine was lost at the Missions by the neophyte's children and grandchildren. 


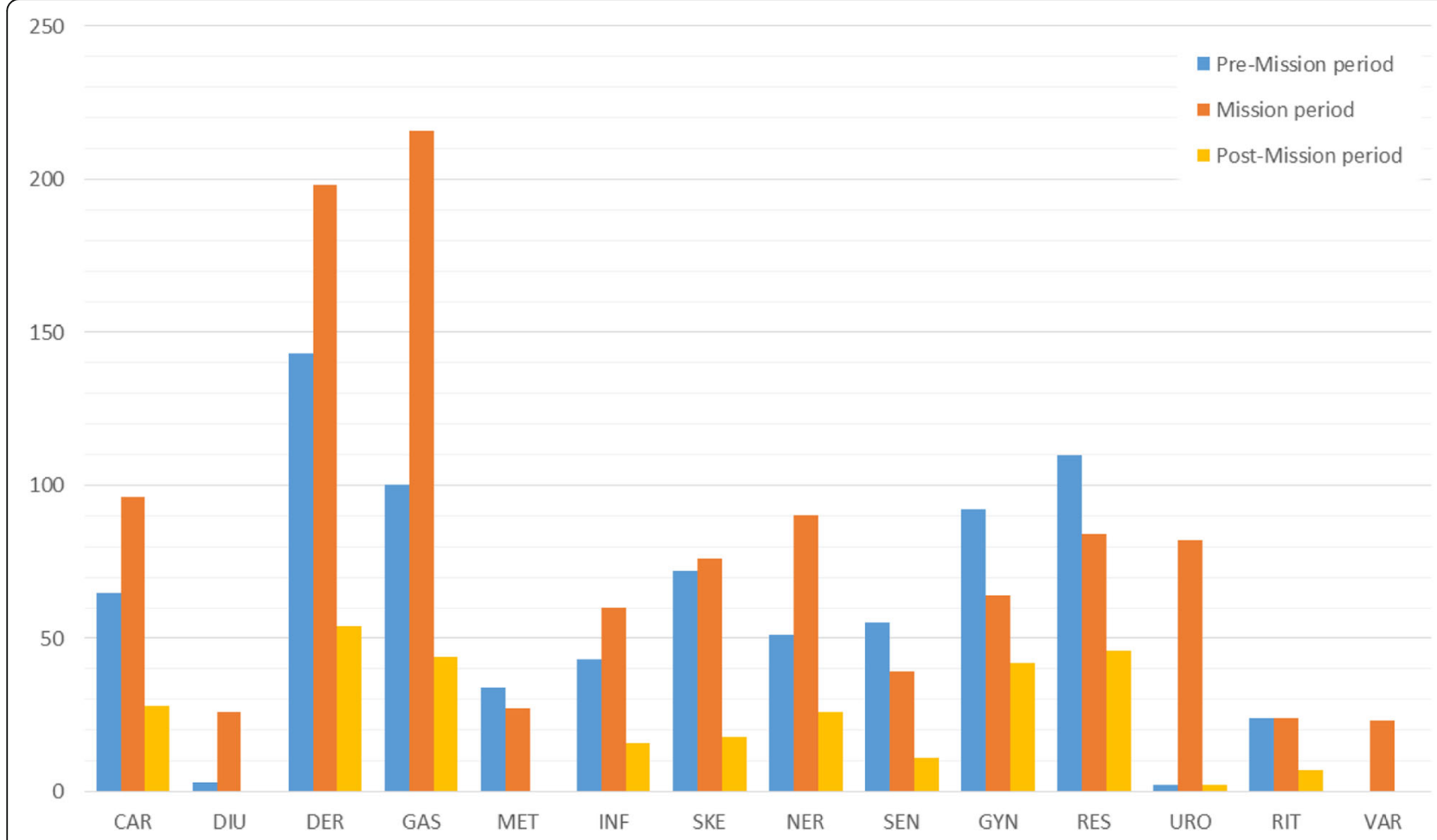

Fig. 2 Therapeutical categories of medicinal plants

(8) Transportation limitations during the Mission period may have limited reciprocal shipments of medicinal plants between Spain and California.

\section{Post-Mission Period}

The list of medicinal plants used both by Natives Americans and Californios indicates a much greater sharing of medicinal knowledge following the secularization of the Missions [19, 43]. The lists indicate 148 taxa were used to treat 288 ailments in 14 therapeutic groups (Fig. 2). Forty-four (30\%) of these 148 taxa occur on the list of medicinal plants used by the Native American prior to the Mission period, forty-two taxa $(28.4 \%)$ were in use during the Mission period.

\section{Discussion}

The results of this study suggest limited sharing of information about medicinal plants occurred during the Mission Period. There are direct reports of the sharing of information such as the dispatching of neophytes to collect food plants and herbs during times of shortages [64]. Additionally, the priest at eight of the Missions responded to the 1812 survey that the local Native Americans used plants for medicinal purposes. One might assume that some of these plants would have been exported to Spain because of their medicinal value. However, none of the 15 species most commonly used by Native Americans occurs on the registry of plants introduced to Mediterranean area during the eighteenth and nineteenth centuries ([48]; Flora [49, 52, 53, 81]). Furthermore, none of these California species were reported to have been grown in present-day herb gardens in northern Spain $[37,38]$. The exchange of information on medicinal plants is further supported by the presence of both European and Californian species in present-day Mission gardens and apothecary shops further supports the exchange of information.

Much more evidence was discovered in this study to suggest many possible factors contributed to constraining the sharing of information about medicinal plants. These factors and the sources of information about these factors are presented in Table 5. We elaborate on these factors as follows:

\section{A significant power imbalance existed between the priests and the Native Americans}

The priests maintained significant power over the Native Americans at the missions. Their power was enforced by corporal punishment and confinement of the neophytes who did not work or who behaved badly in the eyes of the priests $[61,82]$. This power imbalance resulted in the neophytes hiding some information concerning medicinal plants and shaman treating neophytes out of 
Table 4 Published sources supporting the exchange of information on medicinal plants

\begin{tabular}{ll}
\hline 1. Reports of an exchange of information & \\
\hline Comments & Source
\end{tabular}

Native American teach priests about their medicinal plants (pp. 73-74) (example of exchange of information between Native Americans and priests)

Compilation of medicinal plants by Father Garriga (pp. 443-445) (example of exchange of information between Californios and priests)

Father Crespi reports vineyard-like plantings by Native Americans (pp. 45) (example of exchange of information between Native Americans and priests)

Sick sailors taken ashore in hope that medicinal herbs could be found (pp. 143) (example of the use of medicinal plant by Spanish explorers in California)

Dr. Prat searches for medicinal herbs after first ship land in San Diego (pp. 144) (example of the use of medicinal plant by Spanish explorers in California)

List of California plants identified by Portola (pp. 209-293) (example of interest in plants by Spanish explorers)

Native American knowledge of medicinal plants (pp. 66) (example of exchange of information between Native Americans and priests)

Junipero Serra's leg treated by muleteer using local herbs (pp. 69) (example of exchange of information between Mestizo and priests) Friars unable to reduce death rate even with help from Native American shaman (pp. 156) (example of exchange of information between Native Americans and priests)

Dr. Prat searches for medicinal herbs (pp. 14) (example of the use of medicinal plant by Spanish explorers in California)

1812 survey of Missions asking about medicinal practices of Native Americans (example of exchange of information between Native Americans and priests)

Gardens at Mission Delores (pp.58) (example of garden at a Mission where both medicinal plants from Europe and California were grown together for medicinal purposes)

Watercress reported at Mission San Gabriel (pp. 152) (example of medicinal plant native to both Spain and California observed at a Mission) Father Font identifies flora (pp. 176) (example of priest identifying native plants in California and referencing them to plant species in Spain of medicinal value)

Anza becomes sick and is treated with medicinal (pp. 187) (example of exchange of information between Native American and Spanish explorers)

Shared indigenous knowledge (pp. 33) (example of exchange of information between Native Americans and priests)

Neophytes were sometimes dispatched by the priests to collect medicinal plants from the wild (p. 576) (example of exchange of information
Anderson [26]

Beebe and Senkewicz [43]

Blackburn and Anderson [62]

Brown [58]

Boscana [63]

Castillo [59]

Engelhardt [64]

Geiger and Meighan [23]

Goerke [65]

Guerrero [60]

Kryder-Reid [66]

Engelhardt (1922)
Table 4 Published sources supporting the exchange of information on medicinal plants (Continued)

\begin{tabular}{ll}
\hline 1. Reports of an exchange of information & \\
\hline Comments & Source \\
\hline between Native Americans and priests) &
\end{tabular}

At Mission San Jose the Native Americans retained Milliken [67] their native customs (pp. 50-53) (example of $\mathrm{Na}$ tive Americans continuing their use of medicinal plants at the Missions)

Continued practice of native medicine at Soledad Mission (pp. 119) (example of Native Americans continuing their use of medicinal plants at the Missions)

Practice of herbal medicine (pp. 173) (example of Native Americans continuing their use of

medicinal plants at the Missions)

Use of Datura toothache (pp. 175-178) (example of Native Americans continuing their use of medicinal plants at the Missions)

Use of horehound (pp. 180-181) (example of $\mathrm{Na-}$ tive Americans continuing their use of medicinal plants at the Missions)

Gardens at San Buenaventura (pp.86) (example of Native American medicinal plants being planted in Mission gardens)

Exchange of information about medicinal plants (pp.160-161) (example of exchange of information between Native Americans and priests)

\section{Mission gardens and apothecary shops}

Shaman cultivated medicinal herbs (pp. 44) (example of Native American medicinal plants being planted in Mission gardens)

Seed imported from Mexico for Mission gardens (example of plants from a variety of sources being planted in Mission gardens)

San Carlos Mission garden (pp. 186) (example of Native American medicinal plants being planted in Mission gardens)

San Diego Mission gardens (pp. 36) (example of Native American medicinal plants being planted in Mission gardens)

Mission San Buenaventura gardens (pp. 294) (example of Native American medicinal plants being planted in Mission gardens)

San Luis Rey Mission gardens (pp. 96, 98) (example of Native American medicinal plants being planted in Mission gardens)

Native American gardens (pp. 60) (example of Native American medicinal plants being planted in Mission gardens)

Mission San Luis Rey gardens (pp. 76) (example of Native American medicinal plants being planted in Mission gardens)

Domestication of native herbs (pp. 125) (example of Native American medicinal plants being planted in Mission gardens) Apothecary shops (pp. 129-13) (example of Native American medicinal plants being planted in Mission gardens)

Native Americans encouraged to domesticate local plants (pp. 133) (example of Native American medicinal plants being planted in Mission
Blackburn and Anderson [62]

Brown [58]

Guerrero [60]

Kryder-Reid [66]

Lamb [70]

$\operatorname{Tac}[71]$

Webb [61]

Weber [69] 
Table 4 Published sources supporting the exchange of information on medicinal plants (Continued)

\begin{tabular}{l}
\hline 1. Reports of an exchange of information \\
\hline Comments \\
\hline gardens) Specialized gardens at different Missions \\
(pp. 134) \\
Apothecary shops in all Missions (pp. 160) \\
(example of Native American medicinal plants \\
being planted in Mission gardens) \\
\hline
\end{tabular}

sight of the priests $[21,79]$. Any acknowledgment of the value of Native American herbs by the priests would have been a way of giving power to the Native Americans.

\section{Priests thought the Native Americans were savage heathens or children who knew nothing}

Many of the priests regarded the Native Americans as pagan savages whose customs needed to be suppressed. Interest in or communication about native medicinal plants would have been considered a way of endorsing native beliefs that the priests were dedicated to eliminating.

\section{Language barriers to communication}

Language was also a barrier to communication between the priests and the Native Americans. Several quite distinct languages and dialects were spoken by Native Americans living along the California coast. Although the Mission priests were expected to learn the native languages and instruct the Native Americans in their native languages this was seldom the case [59]. The language barrier was limited not only to the difficulty and reluctance of the Mission priests to learn the native languages, but also to the first generation of Native Americans neophytes who learned only a minimum of Spanish. Spanish was acquired by Native Americans born at the Missions [61], but this and subsequent generations of Mission born Native Americans had less knowledge of native medicinal plants to share with the priests.

\section{Reduction in the availability of medicinal herbs due to the elimination of Native American burning and the introduction of Spanish livestock}

The use of land for farming and livestock grazing along with the elimination of Native American burning of the landscape resulted in fewer medicinal plants in the vicinity of the Missions [30, 62, 74]. The resulting lack of access to native medicinal plants further interfered with the transfer on information.
Knowledge of medicinal plants was a source of power and income for the Native American shamans who did not want to share it

The power and income Native American shamans received from their use of medicinal herbs were values that they would not have wanted to give up. The shamans continued their treatment of sick Native Americans at the Missions, but not in situations where they would be observed by the priests ([21, 74]; Timbrook 2000). Since the shaman's knowledge of healing was acquired over many years and was not shared with the general population of Native Americans [80], one would not have expected they would be eager to share it with the priests.

\section{Structural organization of the administration of Missions left little time for direct communication between priests and neophytes}

The Missions were initially organized to be administered by only two priests. They were assisted by a limited number of soldiers, cowboys, farmers, and craftsmen brought from Mexico [77]. Wives of some of these individuals were put in charge of the girl's and unmarried women's dormitories. Others worked as cooks. The priests selected neophytes to serve as acaldes and enfermeros in intermediate positions between the assistants brought from Mexico and the common neophytes [76]. The priests organized the work force of neophytes into four classes: first-skilled artisans; masons, carpenters, etc.; second-fishermen, stockmen, herdsmen, cowboys, tallow makers, hide cleaners, butchers; third-horticulturalists who tended mission gardens; fourth-laborers and field hands [76]. This administrative structure was necessary to manage the large numbers of neophytes at the Mission and to raise food [67]. The administrative structure limited one on one communication between the neophytes and the priests except in the catechism classes initially conducted by the priests. The priests had limited contact with the Native American women, some of whom were lower-level shamans possessing considerable knowledge of medicinal plants [79].

\section{Knowledge of herbal medicine was lost at the Missions} by the neophyte's children and grandchildren

An important impediment to the transfer of knowledge of herbal medicine was the loss of such knowledge by the initial generation of neophyte's children and grandchildren [32, 59, 74]. The individuals who were born at the Missions had fewer contacts with native medicinal plants than Native Americans living away from the Missions. Sandos [76] suggests that previous customs changed the longer the neophytes were at the Missions. 
Table 5 Limitations to the exchange of information on medicinal plants

1. A significant power imbalance existed between the priests and the Native Americans
Comments
The power of the priests was maintained by the presence of soldiers at the missions ( $p$. 22) (example of imbalance of
power between priests and Native Americans)
Priests used corporal punishment to enforce their power (p. 113) (example of imbalance of power between priests and
Native Americans)
Native Americans avoided a sharing of their knowledge of medicinal plants and healing practices by conducting
healing activities at night out of sight of priests from fear of losing power to the priests ( $47-51 ; 71-80,97-100$,
119-120) (example of imbalance of power between priests and Native Americans)
2. Priests thought the Native Americans were savage heathens or children and their pagan ways should be suppressed
Comments

Boscana's view of the character of the Native American (pp. 52) (example of disrespect on the part of priests for Native American knowledge)

Spanish attitude toward Native Americans (pp. 64) (example of disrespect on the part of priests for Native American knowledge)

Fray Lausen's poor view of Native Americans (pp. 93-94) (example of disrespect on the part of priests for Native American knowledge)

Friars harangued Native Americans about their "savage" way of life (pp. 119) (example of disrespect on the part of priests for Native American knowledge)

Boscana referred to shamans as "diabolical imposters" (pp. 236) (example of disrespect on the part of priests for Native American knowledge)

Shamans practiced quackery (pp. 237-238) (example of disrespect on the part of priests for Native American knowledge)

Fr. Boscana's views of Native Americans (example of disrespect on the part of priests for Native American knowledge)

Fundamental duty of missionaries is to eradicate what is harmful in Native American customs (pp. 128-129) (example of disrespect on the part of priests for Native American knowledge)

Spanish hold native culture in contempt (p. 30) (example of disrespect on the part of priests for Native American knowledge)

Priest force Native Americans to alter their traditional practices (pp. 59) (example of disrespect on the part of priests for Native American knowledge)

Shamans considered sorcerers and wizards by priests (pp. 109) (example of disrespect on the part of priests for Native American knowledge)

Controlling and acculturating Native Americans (pp. 110) (example of disrespect on the part of priests for Native American knowledge)

Missionaries sought to make Native Americans ashamed of their traditional ways of life (pp. 223)

Native rituals and beliefs identified as work of the Devil (pp. 225)

Priest have contempt for Native American's abilities (p. 52) (example of disrespect on the part of priests for Native American knowledge)

Priests prohibit Native American from dancing at San Gabriel Mission (pp. 5) (example of disrespect on the part of priests for Native American knowledge)

Fr. Boscana compares Native Americans to monkeys (pp. 21) (example of disrespect on the part of priests for Native American knowledge)

"denaturalizing" of Native Americans (pp. 92) (example of disrespect on the part of priests for Native American knowledge)

Shaman practiced sucking of objects from bodies of the afflicted (pp. 118) (example of disrespect on the part of priests for Native American knowledge)

Tribal lore kept secret by Shaman (pp. 181-182) (example of disrespect on the part of priests for Native American knowledge)

Native Americans viewed as deceivers (pp. 481) (example of disrespect on the part of priests for Native American knowledge)

Native Americans viewed as devil worshipers (pp. 68) (example of disrespect on the part of priests for Native American knowledge)

Challenge to indigenous medicinal practice (pp. 17) (example of disrespect on the part of priests for Native American knowledge)

\section{Language barriers to communication}

Original languages spoken by some neophytes usurped by other languages spoken by neophytes from different tribes (pp.51) (example of disrespect on the part of priests for Native American knowledge)

Native American languages unworthy of study or preservation (pp. 51) (example of disrespect on the part of priests for Native American knowledge)

Widespread lack of Spanish among neophytes (pp. 128a) (example of barrier to sharing of information due to different
Source

Webb [61]

Castillo [59]

Geiger and Meighan [23]

Source

Castillo [59]

Engelhardt [64]

Hanke [72]

Kryder-Reid [66]

Langsdorff [73]

Lightfoot [21]

Milliken [74]

Rawls [75]

Sandos [76]

Shipek [77]

Skowronek [78]

Wilken-Robertson [32]

Castillo [59] 
Table 5 Limitations to the exchange of information on medicinal plants (Continued)

1. A significant power imbalance existed between the priests and the Native Americans

Comments

languages)

No record that teachers were sent or that the friars established to teach Native Americans Spanish (pp. 128b) (example of barrier to sharing of information due to different languages)

Policy of not teaching Native Americans to read or write Spanish (pp. 128-129) (example of barrier to sharing of information due to different languages)

Missionaries did not learn native languages (pp. 140) (example of barrier to sharing of information due to different languages)

Perseverance and hard work required of the missionaries to learn Native American languages (pp. 177) (example of barrier to sharing of information due to different languages)

Missionaries should make greater effort to learn Native American languages (pp. 39) (example of failure of priests to learn native languages)

Language barriers (pp. 26 and 45) (example of barrier to sharing of information due to different languages)

Variety of crude and barbarian languages among the Native Americans (pp. 46) (example of barrier to sharing of information due to different languages)

Native Americans born in the Missions learned Spanish (pp. 47) (example of greater opportunity of second generation neophytes to exchange information on medicinal plants)

Interpreters employed to neophytes since most padres did not learn the native languages (pp. 48a) (example of barrier to sharing of information due to different languages)

Only those Native Americans born in the Mission all speak Castilian (pp. 48b) (example of greater opportunity of second generation neophytes to exchange information on medicinal plants)

After 1840 Native Americans reported to speak Spanish (pp. 308) (example of greater opportunity of second generation neophytes to exchange information on medicinal plants)

Great variety of Native American languages and dialects (pp. 15) (example of greater opportunity of second generation neophytes to exchange information on medicinal plants)

Majority of the friars taught neophytes in Spanish, rather than in their native languages (pp. 124) (example of greater opportunity of second generation neophytes to exchange information on medicinal plants)

\section{Reduction in the availability of medicinal herbs due to the elimination of Native American burning and the introduction of Spanish livestock.}

Subsistence practices constrained at Missions (pp. 79) (example of Native American customs, including medicinal practices constrained at the Missions)

Plant management practices by Native Americans that would have been curtailed around the Missions (pp. 83) (example of Native American customs, including medicinal practices constrained at the Missions)

Native American spiritual practices connected to plant harvesting curtailed by Missionaries (pp. 84) (example of Native American customs, including medicinal practices constrained at the Missions)

Cessation of native fire management practices (pp. 27-28) (example of land management practices used by Native American to promote medicinal plants constrained at the Missions)

Change of lifestyle resulted in a loss of interest in traditional commodities (pp. 222) (example of Native American customs, including medicinal practices constrained at the Missions)

Use of fire by Native Americans (pp. 12) (example of land management practices used by Native American to promote medicinal plants constrained at the Missions)

Spanish soldiers destroy Native American field by grazing (pp. 48-49) (example of land management practices used by Native American to promote medicinal plants constrained at the Missions)

Native American burning to produce more seeds (pp. 81) (example of Native American land management practices used to promote medicinal plants)

Native American burning (pp. 117) (example of Native American land management practices used to promote medicinal plants)

Crespi's observation of Native American burning (pp. 121-122) (example of Native American land management practices used to promote medicinal plants)

Evidence of Native American burning (pp. 124) (example of Native American land management practices used to promote medicinal plants)

Governor Arrillaga bans Native American burning in 1793 (pp. 126-127a) (example of land management practices used by Native American to promote medicinal plants constrained at the Missions)

Moncada's 1774-1777 observations of Native American burning (pp. 126-127b) (example of Native American land management practices used to promote medicinal plants)

Longinos' observation of Native American burning (pp. 129) (example of Native American land management practices used to promote medicinal plants)

Native American use of fire to influence plant growth (pp. 134) (example of Native American land management practices used to promote medicinal plants)

Medicinal plants encouraged by Native American burning (pp. 145) (example of Native American land management practices used to promote medicinal plants)

Adoption of Native Americans to colonist's land management practices (pp. 27) (example of land management practices
Source

Guerrero [60]

Rawl (1984)

Webb [61]

Weber [69]

Lightfoot [21]

Lightfoot and Parrish [30]

Milliken [74]

Timbrook [18]

Blackburn and Anderson [62]

Wilken-Robertson [32] 
Table 5 Limitations to the exchange of information on medicinal plants (Continued)

\section{A significant power imbalance existed between the priests and the Native Americans \\ Comments \\ used by Native American to promote medicinal plants constrained at the Missions) \\ Spanish authorities prohibit Native Americans from burning (pp. 45) (example of land management practices used by \\ Native American to promote medicinal plants constrained at the Missions) \\ 5. Knowledge of medicinal plants was a source of power and income for the Native American shamans who did not want to share it}

Structure of shamanism among California Native Americans (pp. 55-56) (example of Native American power structure effecting the use of medicinal plants)

Secret knowledge (pp. 3) (example of Native American power structure effecting the use of medicinal plants)

Continued native practice of medicine (pp. 110) (example of Native American power structure effecting the use of medicinal plants)

Native practices took place in neophyte quarters (pp. 112-113) (example of Native American power structure effecting the use of medicinal plants)

Priests lament continued pagan practices of shamans at missions (pp. 183) (example of difficulty priest had in curtailing Native American customs)

Shaman's skills required a "lifetime' of experience (pp. 132-133) (example of Native American power structure effecting the use of medicinal plants)

Shaman's methods of healing (pp. 27-28) (example of Native American power structure effecting the use of medicinal plants)

Shamans were skilled at the arts of healing (pp. 10) (example of Native American power structure effecting the use of medicinal plants)

Neophytes preserved much of their culture after baptism without the knowledge of the priests (pp. 94) (example of Native Americans attempting to preserve their knowledge and use of native plants for medicinal purposes)

Different kinds of shamans (pp. 142) (example of Native American power structure effecting the use of medicinal plants) Shamans secretive about their remedies (pp. 173) (example of Native Americans attempting to preserve their knowledge and use of native plants for medicinal purposes)

6. Structural Organization of the administration of Missions left little time for direct communication between priest and neophytes

Alcaldes appointed by priests (pp. 112) (example of priests using intermediaries in dealing with Native Americans)

Priest's organization of neophyte community at the missions (pp. 9) (example of priests using intermediaries in dealing with Native Americans)

Number of Spanish/Mexican people at the mission compared to number of neophytes (pp. 488) (example of the large numbers of Native Americans at themission compared to priest)

\section{Knowledge of herbal medicine lost by the neophyte's children and grandchildren}

Traditional customs forgotten at the missions (pp. 192) (example of knowledge lost by second and third generation neophytes)

Undermining of traditional knowledge from one generation to the next at the missions (pp. 221) (example of knowledge lost by second and third generation neophytes)

Gradual impoverishment of Native American lifestyle at the missions (pp. 222) (example of knowledge lost by second and third generation neophytes)

Previous ways changed the longer neophytes were at the missions (pp. 157) (example of knowledge lost by second and third generation neophytes)

Neophytes lost touch with their culture quickly at the northern mission, but not so quickly at the southern missions (pp. 181-182) (example of knowledge lost by second and third generation neophytes)

Impact of mission system on indigenous medical knowledge (pp. 17) (example of knowledge lost by second and third generation neophytes)

Impact of historical processes on ethnobotanical knowledge (pp. 15-16) (example of knowledge lost by second and third generation neophytes)

\section{Limitations to transportation}

Spanish restriction of exclusion and restriction of foreign trade with their possessions in the New World would have limited the transport of medicinal plants back to Spain (pp. 436-437) (example of constraints on the transportation of medicinal plants)

Every year a transport ship arrived in San Diego, Santa Barbara, Monterey, and San Francisco with supplies for the Missions. Priests were required to pay for and to pay for any materials shipped back to Spain. The costs restricted shipment of medicinal herbs. (pp. 437) (example of constraints on the transportation of medicinal plants) In 1825 Governor Echeandia forbid the missionaries to trade with any vessel outside of the four Presidio ports. This required the expensive transport of materials on the backs of mules from Missions distant
Source

Bean [79]

Boscana [63]

Lightfoot [21]

Margolin [80]

Milliken [74]

Rawls [75]

Sandos [76]

Timbrook [68]

Lightfoot [21]

Sandos [76]

Shipek [77]

Castillo [59]

Milliken [74]

Sandos [76]

Wilken-Robertson [32]

Engelhardt [64] 
Table 5 Limitations to the exchange of information on medicinal plants (Continued)

\begin{tabular}{lc}
\hline 1. A significant power imbalance existed between the priests and the Native Americans & Source \\
\hline Comments & Lightfoot [21] \\
After the ports (pp. 224) (example of constraints on the transportation of medicinal plants) & \\
Mexico. This caused the missions to become more dependent on local landscapes for food & \\
and basic goods (pp. 67) (example of constraints on the transportation of medicinal plants) & Langsdorff (1927) \\
Native Americans received inadequate medical care because of limited supplies of medicines & \\
(pp. 251-252) (example of constraints on the transportation of medicinal plants) &
\end{tabular}

\section{Transportation limitations during the Mission period may have limited reciprocal shipments of medicinal plants between Spain and California}

Transportation from Spain to California and vice versa during the Mission period was limited. Most materials brought from Spain were shipped to ports on the east coast of Mexico, transported over land to Puerto Vallarta, and then shipped to ports in San Diego, Santa Barbara, Monterey, and San Francisco. Occasionally, ships from Europe would travel around the tip of South America to reach ports in California. Prior to the Mexican revolution, at least one ship would arrive annually with supplies for the Missions. During the Mexican War of Independence (1810-1821) shipments to California were for the most part halted [21, 69]. The Spanish priests did import European plants, including medicinal plants for gardens at the Missions [24]; however, observers at the time reported that the Native Americans received inadequate medical care mostly because of limited supplies of medicines [23, 73, 82]. As transportation was limited, especially during the conflict between Spain and Mexico there may have been little opportunity to ship medicinal plants back to Spain or to import them.

A greater exchange of information occurred during the post-Mission Period. The high number of plants used for medicinal purposes might be explained by the closer working relationships that occurred on the local ranches between the Native Americans and the Californios. Furthermore, the Californios had less incentives to "deculturalize" the Native Americans. Preparation of 46 of the herbal remedies reported by Garriga included ingredients (e.g., milk, whisky, castor oil) that were not available to the Native Americans in pre-Spanish times [19]. This suggests a sharing of information between the Californios and the Native Americans. We believe the greater sharing of information about the medicinal use of plants during the secularization and postsecularization period was due to (1) more one-to-one interactions between the Californios and the Native Americans, (2) many of the Californios were mestizos whose mothers or grandmothers were Native Americans, and (3) the lack of pressure on the part of the Californios to suppress Native American beliefs.

\section{Conclusions}

We conclude from this study that there was a limited transfer of information on the medicinal use of plants between the Native American and Spanish priests during the Mission period. Many factors related to the obligations of the priests, their attitudes toward the Native Americans, language barriers, and cultural differences interfered with a more complete sharing of information. A primary factor in the lack of transfer of medicinal information between the Native American and the priest was the imbalance of power. This imbalance of power kept the Native Americans from sharing information. The fact that none of the 15 most commonly used California species were not transported to Spain for medicinal uses presents an interesting question: were these plants not considered of superior value to the plants in Spain for the treatment of illnesses or did the Native American not share their knowledge of these plants with the priests? The magnitude sharing of information about medicinal plants between the Native Americans and the Californios increased in the post-Mission Period. This increase was due to a greater contact between the Native Americans and the Californios and a different relationship that existed between the two groups. Important aspects of this relationship were increased one-on-one communication, mestizo background of the Californios, and the lack of responsibility on the part of the Californios to convert the Native Americans to christianity.

\section{Acknowledgements \\ Not applicable}

\section{Authors' contributions}

McBride, J.-40\% ; Cavero-35\%; Cheshire-10\%; Calvo-10\%; McBride, D.-5\%. JRM initiated the study, developed the research plan, identified references for plants used by Native Americans, developed the lists of medicinal plants from Mexico and medicinal plants used by the Californios and Native Americans during the post-Mission period, conducted the "close reading" of historical and current documents concerning the sharing of medicinal information during the Mission and post-Mission periods in California, and wrote the first draft of the paper. RYC developed data on the use of medicinal plants in Spain, consulted historical sources for introduction of California plants into Spain, served as principal editor of the manuscript, formatted tables, and produced Fig. 2. ALC compiled the list of plant species used by the Native Americans, developed the historical background for the California Missions, and produced Fig. 1. MIC assisted RYC in the development of the lists of medicinal plants used in Spain. DLM provided information on the 
disease epidemics at the California Mission and assisted in the editing of the initial manuscript.

\section{Author's information}

JRM — Plant Ecologist. Professor Emeritus, Department of Environmental Science, Policy, and Management and Department of Landscape Architecture and Environmental Planning, University of California, Berkeley, USA

RYC - Botanist. Professor, Department of Environmental Biology, School of Sciences, University of Navarra.

ALC—Art Historian. Student. School of Art, San Francisco State University, San Francisco, CA.

MIC-Ethnobotanist. Professor. Department of Pharmaceutical Technology and Chemistry, School of Pharmacy and Nutrition, University of Navarra. DLM—Pediatrics. Professor. School of Nursing, Samuel Merritt University, Oakland, CA.

\section{Funding}

This research was funding out of the salaries paid to the authors from their institutions. No direct research funding supported the research and manuscript preparation.

Funding for publication costs will be covered by the University of California (Digital Library 15\%) and personal funds of Joe R. McBride. Any discount would be appreciated.

\section{Availability of data and materials}

All data generated or analyzed during this study are included in this published article.

\section{Ethics approval and consent to participate}

No ethics approval was required for this research at any of the Universities where the authors worked because the research did not involve human subjects, live animals, or live plants.

\section{Consent for publication}

Not applicable

\section{Competing interests}

There are no financial and non-financial competing interests involved in this research or its publication.

\section{Author details}

'Department of Environmental Science, Policy and Management, University of California, Berkeley, CA, USA. ${ }^{2}$ Department of Environmental Biology, School of Sciences, University of Navarra, Irunlarrea 1, 31008 Pamplona, Spain. ${ }^{3}$ School of Art, San Francisco State University, San Francisco, CA, USA. ${ }^{4}$ Department of Pharmaceutical Technology and Chemistry, School of Pharmacy and Nutrition, University of Navarra, Irunlarrea 1, 31008 Pamplona, Spain. ${ }^{5}$ School of Nursing, Samuel Merritt University, Oakland, CA, USA.

\section{Received: 2 February 2020 Accepted: 3 June 2020}

\section{Published online: 15 June 2020}

\section{References}

1. Bonatto SL, Salzano FM. A single and early migration for the peopling of the Americas supported by mitochondrial DNA sequence data. PNAS. 1997; 94:1866-71.

2. Jones TL, Fitzgerald RT, Kenneth DJ, Miksicek CH, Fagan JL, Sharp J, Erlandson JM. The cross creek site (CA-SLO-1979) and its implications for new world colonization. Am Antiq. 2002;67:213-30.

3. Codding BF, Jones TL. Environmental productivity predicts migration, demographic, and linguistic patterns in prehistoric California. PNAS. 2013; 110:14569-73.

4. Verma R, Gangrade T, Punasiya R, Ghulaxe C. Rubus fructicosus (blackberry) use as an herbal medicine. Phcog. Rev. 2014;8:101-4.

5. Dixon RB. The Northern Maidu. Bull. Am. Mus. Nat. Hist. 1905;17:119-346.

6. Barrett SA. The Ethno-Geography of the Pomo and neighboring Indians. Berkeley: University of California; 1908.

7. Bard CL. A contribution to the history of medicine in southern California, Annual Address of the Retiring President of the Southern California Medical. San Diego: Society; 1894.
8. Bean LJ, Saubel KS. Temalpakh: Cahuilla Indian knowledge and usage of plants. Morongo Indian Reservation: Malki Museum Press; 1972.

9. Garcia C, Adams JD. Healing with medicinal plants of the west - cultural and scientific basis for their use. La Crescenta: Abedus Press; 2005.

10. Gardner L. The surviving Chumash. Archaeol. Survey Annu. Univ. California. 1965;7:277-302.

11. Mead GR. The ethnobotany of the California Indians: a compendium of the plants, their users, and their uses. Museum of Anthropology: University of Northern Colorado, Greeley; 1972.

12. Moerman DE. Native American Ethnobotany. Portland: Timber Press; 2000.

13. Murphey EVA. Indian uses of native plants. Fort Bragg: Mendocino County Historical Society; 1959.

14. Sparkman PS. Culture of the Luiseno Indians. American Archaeology and Ethnology. 1908;8:187-234.

15. Strike SS. Aboriginal uses of California's indigenous plants, ethnobotany of the California Indians, vol. 2. Champaign: Koeltz Scientific Books; 1994.

16. Timbrook J. Virtuous herbs: plants in Chumash medicine. J. Ethnobiology. 1987;7:171-80.

17. Timbrook J. Ethnobotany of Chumash Indians, California, based on collections by John P. Harrington. Economic Botany. 1990;44:236-53.

18. Timbrook J. Chumash ethnobotany: plant knowledge among the Chumash people of Southern California. Berkeley: Heyday Books; 2007.

19. Weber FJ, editor. Andrew Garriga's compilation of herbs and remedies used by the Indians \& Spanish Californians. Los Angeles: Archdiocese of Los Angeles; 1978.

20. Heizer RF, editor. Handbook of North American Indians. Vol. 8, California. Washington: Smithsonian Institution; 1978.

21. Lightfoot KG. Indians, missionaries, and merchants. Berkeley: University of California Press; 2005.

22. Geiger M. Biographical data on the missionaries of San Fernando College serving the California Missions from 1817 to 1820 . Calif. Hist. Soc. Q. 1969;48: 125-51.

23. Geiger M, Meighan CW. As the padres saw them. In: California Indian life and customs as reported by the Franciscan Missionaries 1813-1815. Santa Barbara Mission Archive Library: Santa Barbara; 1976.

24. Dunmire WW. Gardens of New Spain. Austin: University of Texas Press; 2004

25. Cook SF. Population trends among the California Mission Indians. Berkeley: University of California Press; 1967.

26. Anderson MK. Tending the wild. Berkeley: University of California Press; 2005.

27. Barrows DP. The ethnobotany of the Coahuilla Indians of Southern California. Chicago: University of Chicago Press; 1909.

28. Faber G, Lasagna M. Whispers from the first Californians. Alamo, CA: Mabpie Publications; 1980

29. Heinsen V. Mission San Antonio de Padua Herbs. Jolon: Printed by Old Mission San Antonio; 1989.

30. Lightfoot KG, Parrish O. California Indians and their environment: an introduction. Berkeley: University of California Press; 2009.

31. Mead GR. The ethnobotany of the California Indians. La Grange: E-Cat Worlds Press; 2014.

32. Wilken-Robertson M. Kumeyaay Ethnobotany. Inc, San Diego: Sunbelt Publications; 2018

33. Akerreta S, Cavero RY, Calvo MI. First comprehensive contribution to medical ethnobotany of Western Pyrenees. J. Ethnobiol. Ethnomed. 2007a;6: 26-39.

34. Akerreta S, Cavero RY, López V, Calvo MI. Analyzing factors that influence the folk use and phytonomy of 18 medicinal plants in Navarra. J. Ethnobiol. Ethnomed. 2007b;3:16.

35. Alarcón R, Pardo-de-Santayana M, Priestley C, Morales R, Heinrich M. Medicinal and local food plants in the south of Alava (Basque Country, Spain). J. Ethnopharmacol. 2015;176:207-24.

36. Carrió, E., Vallès, J., 2012. Ethnobotany of medicinal plants used in Eastern Mallorca (Balearic Islands, Mediterranean Sea). 141, 1021-1040.

37. Cavero RY, Akerreta S, Calvo MI. Pharmaceutical ethnobotany in northern Navarra (Iberian Peninsula). J. Ethnopharmacol. 2011a;133: $138-46$.

38. Cavero RY, Akerreta S, Calvo MI. Pharmaceutical ethnobotany in the middle Navarra (Iberian Peninsula). J. Ethnopharmacol. 2011b;137:844-55.

39. Menendez-Baceta, G., Aceituno-Mata, L., Molina, M., Reyes-García, V., Tardío, J., Pardo-de- Santayana, M., 2014. Medicinal plants traditionally used in the northwest of the Basque Country (Biscay and Alava), Iberian Peninsula. J. Ethnopharmacol. 152, 113-134. 
40. Argueta A, Gallardo VM. Handbook of traditional Mexican medicine. Mexico City: Instituto Nacional Indigenista; 1994.

41. Heinrich M, Ankle A, Frei B, Weimann C, Sticher O. Medicinal plants of Mexico. So. Sci. Med. 1998:47:1863-75.

42. Simpson LB. California in 1792. The expedition of Jose Longinos Martinez. San Marino: Huntington Library; 1938.

43. Beebe, R.M., Senkewicz R.M. (eds.), 2001. Lands of promise and despair: chronicles of early California, 1535 -1846, Heyday Books, Berkeley.

44. Staub PO, Geck MS, Weckerle CS, Casu L, Leonti M. Classifying diseases and remedies in ethnomedicine and ethnopharmacology. J. Ethnopharmacol. 2015;174:514-9.

45. Hickman JC. The Jepson Manual. Berkeley, CA: University of California; 1993.

46. Stevens, P. F. (2001 onwards). Angiosperm phylogeny website. Version 14 July 68 [and more or less continuously updated since]. http://www.mobot. org/MOBOT/research/APweb/.

47. The International Plant Names Index, 2019. https://www.ipni.org/ ().

48. Coste M. Flore descriptive et illustrée de la France, de la Corse et des contrèes limitrophes, vol. I-III. Albert Blanchard, Paris: Librairie Scientifique et Technique; 1937.

49. Font Quer P. Plantas medicinales: el Dioscórides renovado, ed. Barcelona: Península; 1999

50. IECTB. Inventario Español de los Conocimientos Tradicionales Relativos a la Biodiversidad. Alimentación y Medio Ambiente: Ministerio de Agricultura; 2014.www.mapama.gob.es/inventario_esp_conocimientos_tradicionales. $\operatorname{aspx}($.

51. Lozoya, X., 1999. Fuentes del siglo XVI. Libellus de Medicinalibus Indorum Herbis (Librito de las yerbas medicinales de los indios) o Códice Badiano. Arqueología Mexicana 7, 22.

52. Pignatti S. Flora d' Italia, vol. I-III. Bologne: Edagricole; 1982.

53. Tutin TG, Heywood VH, Burges NA, Valentine DH, Walters SM, Webb DA. Flora Europaea, vol. I-V. Cambridge: Cambridge University Press; 1964-1980.

54. Viesca-Treviño C. Uso de las plantas medicinales mexicanas. Arqueología Mexicana. 1999;7:30-5

55. Med-checklist, 2019. http://ww2.bgbm.org/mcl/ ().

56. UNIKA, 2019. http://www.unav.es/biblioteca/unika/informacion.html ().

57. Wikilengua. Plantae Mundi, 2019. http://www.wikilengua.org/index.php/ (accessed 14 March 2019).

58. Brown, T., 1988. Gardens of the California Missions. Pacific Horticulture. https://www.pacifichorticulture.org/articles/gardens-of-the-californiamissions/ ().

59. Castillo E. A cross of thorns. The enslavement of California's Indians by the Spanish Missions: Craven Street Books, Fresno; 2015.

60. Guerrero V. The Anza trail and the settling of California. Berkeley: Heyday Books; 2006.

61. Webb EB. Indian life at the old missions, W. Los Angeles: F. Lewis; 1952.

62. Blackburn TC, Anderson MK, editors. Before the wilderness: environmental management by native Californians, Ballena Press, Anthropological Papers 40. Menlo: Park; 1993.

63. Boscana FG. Chinigchinich, forgotten books. San: Bernardino; 1846.

64. Engelhardt Z. The missions and missionaries of California. San Francisco, CA: James H. Barry Company; 1912

65. Goerke B. Chief Marin. Berkeley: Heyday Books; 2007.

66. Kryder-Reid E. California Mission landscapes. Minneapolis: University of Minnesota Press; 2016.

67. Milliken R. Native Americans at mission San Jose. Banning: Malki-Ballena Press; 2008.

68. Timbrook, J., 2000. Virtuous herbs: plants in Chumash medicine, in: Walker, P.L., Hudson, T. 1993 Chumash healing: changing health and medical practices in an American Indian Society, pp.161.

69. Weber FJ. The California Missions. Strasbourg: Editions du Signe; 2007.

70. Lamb WK, editor. The voyage of George Vancouver, 1771-1775. New York, Routledge: Vol. II; 1984

71. Tac P, Hewes M, Hewes G. Life and customs at Mission San Luis Rey. The Americas. 1952:9:87-106.

72. Hanke, L., 1969. Indians and Spanish in the new world: a personal view, in Peckham, H.,Hardwick, M.R., 2005. Changes in landscape, the beginnings of horticulture in the California Missions, The Paragon Agency, Orange.

73. Langsdorf, G.H. von., 1927. Landgsdorff's narrative of the Rezanov voyage to Nueva California in 1806, Private Press of T. C. Ruddell, Larkspur.

74. Milliken, R., 1995. A time of little choice. Ballena Press Anthropological Paper No. 43, Ballena Press, Menlo Park.
75. Rawls JJ. Indians of California. The Changing Image: University of Oklahoma Press, Norman; 1984

76. Sandos JA. Converting California. New Haven: Yale University Press; 2004.

77. Shipek FC. California Indian reaction to the Franciscans. The Americas. 1985; 41:480-92.

78. Skowronek RK. Telling the Santa Clara story: Sesquicentennial Voices. Santa Clara: Santa Clara University Press; 2002.

79. Bean LJ. California Indian Shamanism. Menlo Park: Ballena Press; 1992

80. Margolin M. The Ohlone way: Indian life in the San Francisco-Monterey Bay area. Berkeley: Heyday Press; 1978.

81. Flora Iberica, 1986-2005. Real Jardín Botánico de Madrid. CSIC. http://www. floraiberica.es (acceded 13 March 2019).

82. Archibald R. Indian labor at the California Missions: slavery or salvation? SDHS/J. 1978;24:172-82.

\section{Publisher's Note}

Springer Nature remains neutral with regard to jurisdictional claims in published maps and institutional affiliations.
Ready to submit your research? Choose BMC and benefit from:

- fast, convenient online submission

- thorough peer review by experienced researchers in your field

- rapid publication on acceptance

- support for research data, including large and complex data types

- gold Open Access which fosters wider collaboration and increased citations

- maximum visibility for your research: over $100 \mathrm{M}$ website views per year

At BMC, research is always in progress.

Learn more biomedcentral.com/submissions 